Aus der Abteilung Palliativmedizin

(Prof. Dr. med. F. Nauck)

im Zentrum Anaesthesiologie, Rettungs- und Intensivmedizin der Medizinischen Fakultät der Universität Göttingen

\title{
Ausmaß und therapeutische Relevanz nichtinvasiver Diagnostik in der Palliativmedizin
}

\author{
INAUGURAL-DISSERTATION \\ zur Erlangung des Doktorgrades \\ der Medizinischen Fakultät der \\ Georg-August-Universität zu Göttingen
}

vorgelegt von

Ruth Maria Sürig

aus

Düren

Göttingen 2011 
Dekan: Prof. Dr. med. C. Frömmel

I. Berichterstatter: Prof. Dr. med. F. Nauck

II. Berichterstatter: Prof.Dr.med.Dr.med.dent.W.Engelke

III. Berichterstatter: Prof.Dr.med.Dr.rer.nat.H.Eiffert

Tag der mündlichen Prüfung: 05.12.2011 


\section{Inhaltsverzeichnis}

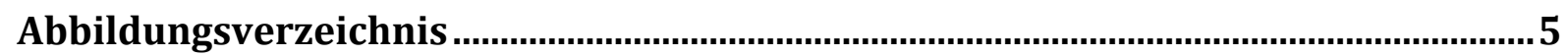

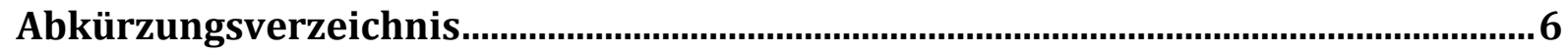

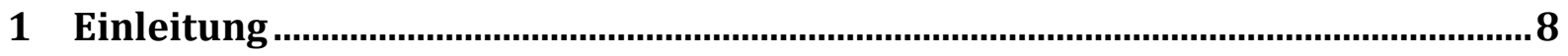

1.1 Entwicklung der Palliativmedizin ........................................................................................

1.2 Begriffserklärungen und Definitionen..................................................................................... 10

1.3 Therapieziele und Diagnostik in der Palliativmedizin .............................................................. 13

1.4 Hospiz- und Palliativerhebung (HOPE) - Patientenregister und Forschungsinstrument14

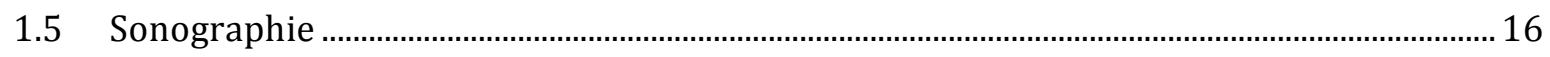

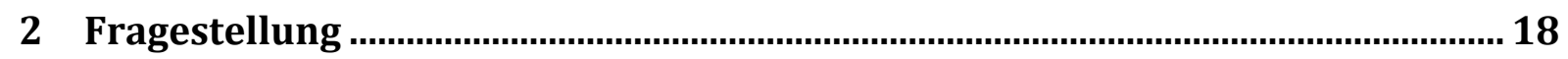

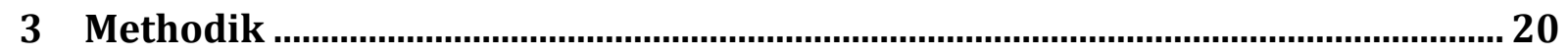

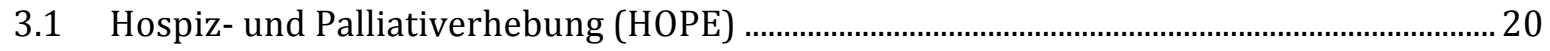

3.2 Abteilungsinterne Dokumentation ……………...................................................................... 23

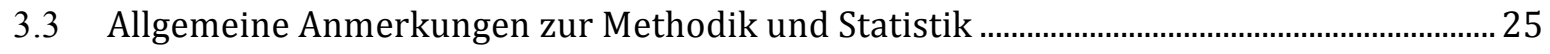

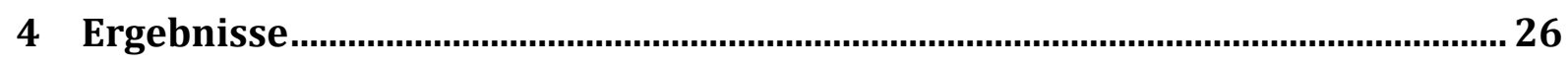

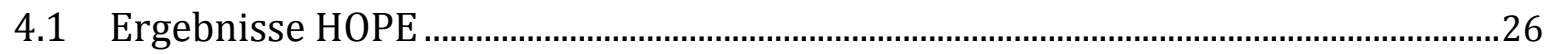

4.1.1 Teilnehmende Einrichtungen an HOPE und DIA-Modul-Rücklauf der HOPE-

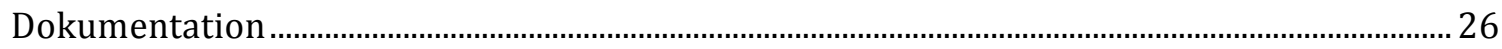

4.1.2 Demographische Daten und Verweildauer des Gesamtkollektivs HOPE....................... 27

4.1.3 Demographische Daten und Verweildauer der HOPE-Patienten mit DIA-Modul...... 27

4.1.4 Auswertung der Rücksendung des DIA-Moduls .................................................................. 28

4.1.5 Auswertung DIA-Modul Teil I: Mikrobiologische Untersuchungen (DI1a.-DI2) ........ 29

4.1.6 Auswertung DIA-Modul Teil II: Bildgebende Verfahren (DI3a.-DI8.) ............................. 30

4.1.7 Auswertung DIA-Modul Teil II: Probleme bei der Durchführung / Beurteilbarkeit 32

4.1.8 Auswertung DIA-Modul Teil I + II: „technisch-analytisches Ergebnis“ “......................... 34

4.2 Datenauswertung der Palliativstation der UMG ……...................................................35

4.2.1 Demographische Daten und Verweildauer der Patienten der Palliativstation

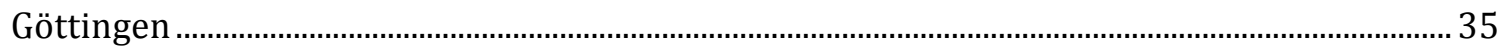

4.2.2 Auswertung der Sonographieergebnisse der UMG-Patienten........................................... 36 


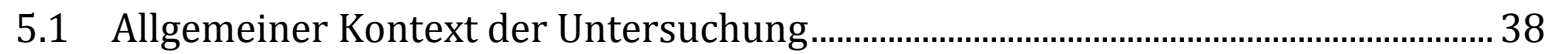

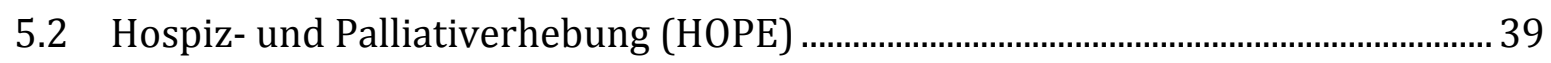

5.2.1 HOPE als Patientenregister und Qualitätssicherungsinstrument.................................... 39

5.2.2 Teilnehmende Einrichtungen an HOPE und Nutzer des Moduls Diagnostik (DIA)... 39

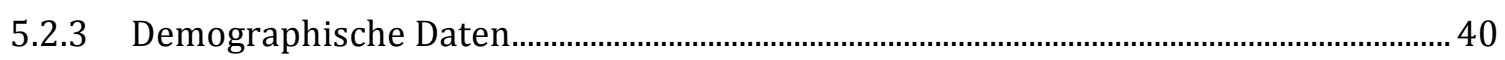

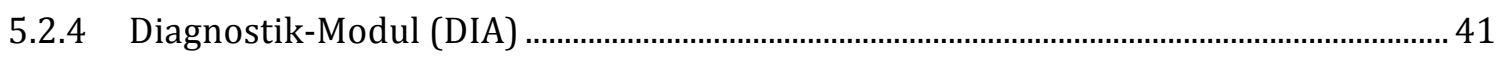

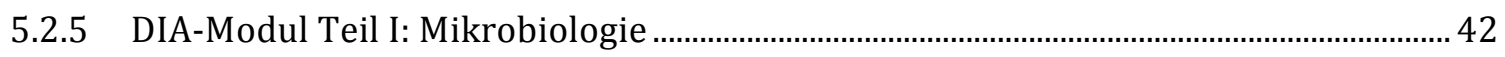

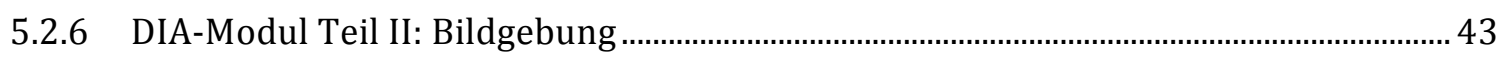

5.2.7 DIA-Modul Teil II: Probleme bei der Durchführung und Beurteilung ............................. 45

5.2.8 DIA-Modul I+II: „technisch-analytisches Ergebnis“ .......................................................... 45

5.3 Sonographien der Palliativstation der Universitätsmedizin Göttingen................... 46

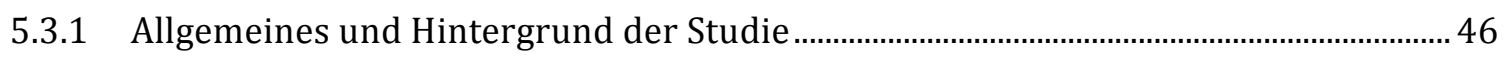

5.3.2 Demographische Daten und Verweildauer der UMG-Patienten ....................................... 47

5.3.3 Auswertung der UMG-Patienten............................................................................................ 47

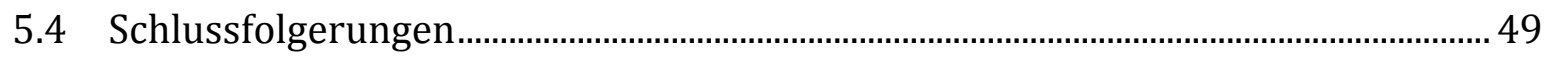

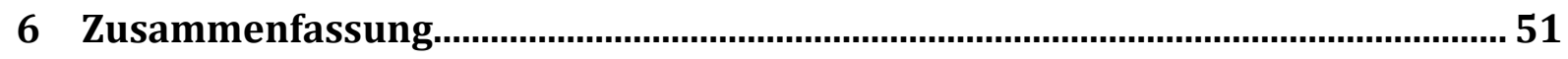

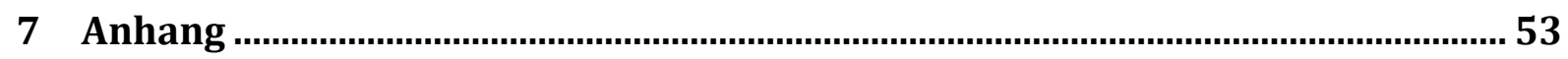

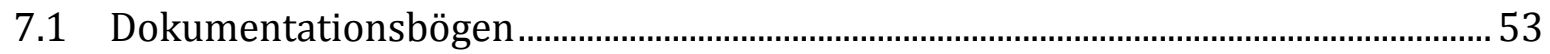

7.1.1 Hospiz- und Palliativerhebung (HOPE) Basisbogen ............................................................. 53

7.1.2 Hospiz- und Palliativerhebung (HOPE) Modul Diagnostik ............................................... 55

7.2 Hospiz- und Palliativerhebung (HOPE) Patienteninformation und

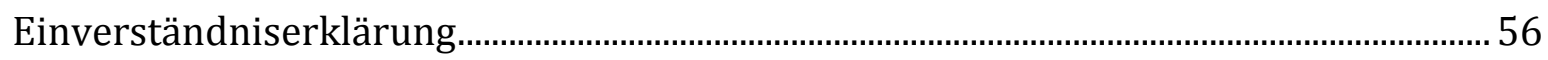

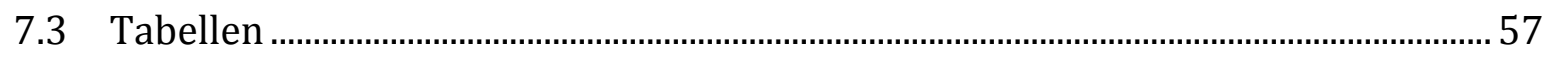

7.3.1 Tabelle von der Homepage der DGP- Kurzinformationen HOPE 2008 ........................ 57

7.3.2 Frequenzen der Sonographie im hausärztlichen Bereich ................................................. 58

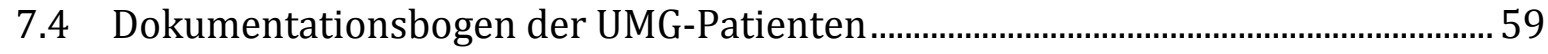

7.5 Englischsprachige Definition „Palliative care“ gemäß WHO

(http://www.who.int/cancer/palliative/definition/en/ 2009): 60

8 Literaturverzeichniss 


\section{Abbildungsverzeichnis}

Tabelle 1: Grundsätze der Palliativmedizin .................................................................................. 8

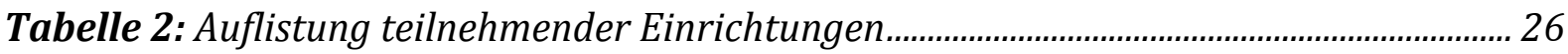

Tabelle 3: Gegenüberstellung der demographischen Daten und der Verweildauer der

Patienten mit DIA-Modul zum Gesamtkollektiv HOPE ............................................................... 28

Tabelle 4: Verteilung der einzelnen Verfahren im DIA-Modul .................................................. 28

Tabelle 5: Auflistung der therapeutischen Konsequenzen ........................................................... 29

Tabelle 6: Prozentuale Verteilung der einzelnen Verfahren ..................................................... 31

Tabelle 7: Aus bildgebender Diagnostik resultierende therapeutische klinische

Konsequenzen

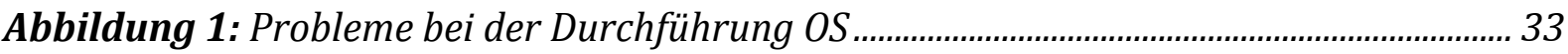

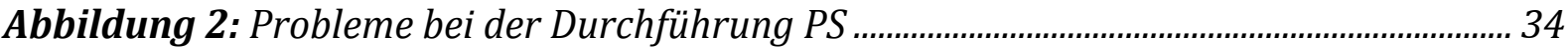

Tabelle 8: Ergebnisse „technisch/analytisches Ergebnis“......................................................... 35

Abbildung 3: Anteile der einzelnen Verfahren an den Konsequenzen...................................... 37

Tabelle 9: Teilnehmende Einrichtungen und Anzahl der dokumentierten Patienten in HOPE im Zeitraum 1999 - 2008. 


\section{Abkürzungsverzeichnis}

- $\mathrm{AA}$

- Abb.

- abs.

- $\mathrm{AP}$

- $\mathrm{BAG}$

- bzw.

- ca.

- ClARA

- $\mathrm{CT}$

- d

- DGP

- d.h.

- DHPV

- DIA

- DKG

- Dr. med.

- EAPC

- evtl.

- $\mathrm{G}$

- $\mathrm{H}$

- HOPE

- KBV

- li.

- männl.

- MIDOS

- mind.

- MRT

- NUK
Ambulanter Arzt

Abbildung

absolut

Ambulante Pflege

Bundesarbeitsgemeinschaft Hospiz

beziehungsweise

circa

Clinical Analysis, Research and Application

Computertomographie

days/ Tage

Deutsche Gesellschaft für Palliativmedizin

das heißt

Deutscher Hospiz- und PalliativVerband

Diagnostik

Deutsche Krebsgesellschaft

Doktor der Medizin

European Association for Palliative Care

eventuell

Geriatrische Station

stationäres Hospiz

Hospiz- und Palliativerhebung

Kassenärztliche Bundesvereinigung

linke

männlich

Minimales Dokumentationssystem

mindestens

Magnetresonanztomographie

Nuklearmedizin 
- OS

- PoBaDo

- POS

- Prof.

- PS

- re.

- RKI

- $\operatorname{sog}$.

- sonst.

- SPSS

- s.u.

- Tab.

- $\mathrm{TT}$

- u.a

- $\mathrm{UMG}$

- UKG

- usw.

- vgl.

- vs.

- WHO

- weibl.

- z.B
Onkologische Station

Psychoonkologische Basisdokumentation

Palliative Care Outcome Scale

Professor

Palliativstation

rechte

Robert- Koch- Institut

so genannte(r/s)

sonstige $(r / s)$

Statistik- Programm- System für Sozialwissenschaften

siehe unten

Tabelle

Tumor(spezifische) Therapie

und andere

Universitätsmedizin Göttingen

Universitätsklinikum Göttingen

und so weiter

vergleiche

versus

World Health Organization

weiblich

zum Beispiel 


\section{Einleitung}

\subsection{Entwicklung der Palliativmedizin}

Das 1967 in London eröffnete St. Christopher's Hospice wird als das weltweit erste Hospiz der modernen Hospiz- und Palliativbewegung angesehen (Twycross, 1980). Gegründet wurde es von Cicely Saunders, die als wichtigste Vorreiterin und Vordenkerin der heutigen Palliativmedizin und Hospizarbeit gilt. C. Saunders formulierte 1977 folgende Grundsätze der Palliativmedizin:

- Behandlung des Patienten in der Umgebung seiner Wahl (ambulant, stationär, zu Hause, Pflegeheim etc.)

- Beachtung der physischen, psychischen, sozialen und seelsorgerischen Bedürfnisse von Patienten, Angehörigen und dem Behandlungsteam

- "high person, low technology"

- individuelle Behandlung jedes Patienten im multidisziplinären Team rund um die Uhr

- Offenheit und Wahrhaftigkeit als Grundlage des Vertrauensverhältnisses unter allen Beteiligten

- Symptomkontrolle (Schmerzen und andere Symptome) durch den Spezialisten (intensive medizinische Betreuung)

- fachliche Pflege durch speziell geschulte Pflegekräfte

- Integration von Ehrenamtlichen

- zentrale Koordination des Teams

- kontinuierliche Betreuung (24-Stunden-Bereitschaft) des Patienten und seiner Angehörigen bis zum Tod bzw. in der Trauerzeit

- Bejahung des Lebens. Akzeptanz von Sterben und Tod als Teil des Lebens. Der Tod wird weder beschleunigt noch hinausgezögert. Aktive Sterbehilfe wird strikt abgelehnt.

- Forschung, Dokumentation und Auswertung der Behandlungsergebnisse

- Unterricht und Ausbildung von Ärzten, Pflegekräften, Sozialarbeitern, Seelsorgern und Ehrenamtlichen

Tabelle 1: Grundsätze der Palliativmedizin (Saunders, 1977) 
Von da an entwickelten sich in Großbritannien die Hospizidee und die Palliativmedizin rasch weiter (Twycross, 1980). Erst 16 Jahre nach 1977 wurde in Deutschland die erste Palliativstation mit Hausbetreuungsdienst eröffnet. Sie entstand durch Prof. Pichlmeier als Förderprojekt der Deutschen Krebshilfe in Köln an der Chirurgischen Universitätsklinik (Klaschik et al., 2000; Sabatowski et al., 2001). Die Entwicklung verlief anfangs langsam, 1990 existierten erst 3 Palliativstationen, erst nachdem 1991 durch das Ministerium für Gesundheit in jedem Bundesland eine Palliativstation gefördert wurde, kam sie relativ schnell voran. Im Jahre 2000 existierten bereits 62 deutsche Palliativstationen, davon waren fünf Universitätskliniken angegliedert (Klaschik et al., 2000; Sabatowski et al., 2001). Zum Untersuchungszeitpunkt im Jahre 2007 gab es bundesweit bereits 142 Palliativstationen (Deutsche Gesellschaft für Palliativmedizin, 2007).

Im Vergleich entwickelte sich die palliativmedizinische Aus-, Fort- und Weiterbildung in Deutschland wesentlich langsamer und noch immer unzureichend (Röglin, 1998; Sabatowski et al., 2001). Der erste Lehrstuhl für Palliativmedizin wurde 1999 in Bonn eingerichtet, weitere folgten in Köln, Aachen, München und Göttingen (Dietz, 2009). Im Jahr 2002 wurde Palliativmedizin als fakultatives Fach in die Approbationsordnung aufgenommen und erst im Jahr darauf (2003) führte die Deutsche Ärztekammer (DÄK) die Zusatzbezeichnung Palliativmedizin für Fachärzte ein. Im Juni 2009 wurde Palliativmedizin als Pflichtlehr- und Prüfungsfach in die ärztliche Approbationsordnung aufgenommen (Nauck, 2009). Auch wenn es durch die beeindruckende Zunahme palliativmedizinischer stationärer Einrichtungen (Radbruch et al., 2002) und die Einführung von einheitlichen Dokumentations- und Qualitätssicherungssystemen in den letzten Jahren $\mathrm{zu}$ einer deutlichen Entwicklung und Verbesserung in der palliativmedizinischen Aus-, Fort- und Weiterbildung für Ärzte und Studenten gekommen ist (Klinkhammer, 2007), bestehen noch immer Defizite (Klaschik, 2001; Lang et al., 2006; Nauck und Radbruch 2002; Nauck et al., 2008).

Auch heute noch wird Palliativmedizin als ,junge Disziplin mit großem Potential“ mit rasanter Entwicklung bezeichnet (Harstensstein, 2002; Klinkhammer, 2007). 


\subsection{Begriffserklärungen und Definitionen}

Bei der Behandlung von Krankheiten unterscheidet man zwei unterschiedliche Therapieansätze: die kurative Therapie und die palliative Behandlung. Die kurative Medizin legt ihren Schwerpunkt auf die "Heilung" (curare, lat. = heilen) und Lebenserhaltung bzw. -verlängerung. Es werden Therapien und Nebenwirkungen toleriert, die oft mit einer erheblichen Verschlechterung und Einschränkung der Lebensqualität des Patienten einhergehen. Die Palliativmedizin hingegen befasst sich nicht mit dem Heilen von Krankheiten, sondern mit dem "Schutz“ („pallium“, der Mantel) des Patienten, der Linderung seiner Symptome („palliare“, lindern), dem Erhalt seiner bestmöglichen Funktionsfähigkeit und seiner Lebensqualität/Zufriedenheit, wenn keine Heilung mehr möglich ist. Oft sind die Übergänge von kurativer zu palliativer Behandlung fließend. Im angelsächsischen Sprachgebiet differenziert man zwischen „Palliative Care“ und „Palliative Medicine“. „Palliative Medicine“ beschreibt im angelsächsischen Sprachgebiet die ärztlichen Maßnahmen und kann definiert werden als „Untersuchungen und Behandlungen von Patienten mit weit fortgeschrittenen Erkrankungen und eingeschränkter Lebenserwartung, bei denen der Schwerpunkt der Behandlung die Lebensqualität ist" (Doyle et al., 2005). Unter „Palliative Care“ hingegen versteht man die "Palliative Betreuung“ des Patienten mit allen nur möglichen multidisziplinären Versorgungsmöglichkeiten (Ärzte, Pflegepersonal, Sozialarbeitern, Psychologen usw.). Da im deutschen Sprachgebrauch die Trennung dieser beiden Begriffe nicht existiert, ist „Palliativmedizin“ mit „Palliative Care" gleichzusetzen (DGP, 2003). Im Folgenden werden die Begrifflichkeiten kurz erörtert:

\section{Palliativmedizin:}

In der Literatur findet man viele Definitionen des Begriffs Palliativmedizin, im Grunde aber sind sie sich alle sehr ähnlich und treffen die gleichen Kernaussagen.

\section{Definition der World Health Organization (1990):}

"Palliativmedizin ist die aktive, ganzheitliche Behandlung von Patienten mit einer progredienten, weit fortgeschrittenen Erkrankung und einer begrenzten Lebenserwartung zu der Zeit, in der die Erkrankung nicht mehr auf eine kurative Behandlung anspricht und die Beherrschung der Schmerzen, anderer 
Krankheitsbeschwerden, psychologischer, sozialer und spiritueller Probleme höchste Priorität besitzt." (World Health Organisation 1990, vgl. Anhang 7.5 aktuelle und erweiterte Definition englische Version)

\section{Definition der European Association for Palliative Care (2004):}

Palliativmedizin ist eine aktive, ganzheitliche Betreuung von Patienten, die an einer unheilbaren Erkrankung leiden. Bei der palliativen Behandlung stehen die Symptomkontrolle, die Schmerztherapie und die psycho-soziale Betreuung der Patienten im Vordergrund. Mit ihrem interdisziplinären Ansatz erfasst die Palliativmedizin nicht nur den Patienten, sondern auch seine Familie und sein soziales Umfeld. Dem Patienten soll die seinen Bedürfnissen angepasste bestmögliche ambulante oder stationäre Betreuung und Versorgung geboten und ermöglicht werden. Die Palliativmedizin bejaht das Leben und sieht das Sterben als einen natürlichen Prozess des Lebens. Sie will diesen weder aufschieben noch herauszögern. Ihr Ziel ist es, dem Patienten die bestmögliche Lebensqualität bis zum Tod zu ermöglichen.

(www.eapcnet.org/about/definition.html)

\section{Definition der Deutschen Gesellschaft für Palliativmedizin (2007):}

„Palliativmedizin ist die Behandlung von Patienten mit einer nicht heilbaren progredienten und weit fortgeschrittenen Erkrankung mit begrenzter Lebenserwartung, für die das Hauptziel der Begleitung die Lebensqualität ist." (www.dgpalliativmedizin.de)

Nach den oben aufgeführten Definitionen beschäftigt sich die Palliativmedizin nicht nur mit der Finalphase, sondern auch mit früheren Krankheitsphasen (Rehabilitations-, Präterminal- und Terminalphase) (Jonen-Thielemann, 2007). Somit ergeben sich ganzheitliche, multidisziplinäre und multizentrische Aufgaben der Palliativmedizin. Das Hauptziel der Palliativmedizin ist die Verbesserung der Lebensqualität für den Patienten und seine Angehörigen (www.who.int).

Um dieses Ziel zu erreichen, umfasst die Palliativmedizin, wie auch im Curriculum Palliativmedizin aufgeführt, folgende Inhalte und Prinzipen (Müller et al., 1997): 
1. physische Aspekte (medikamentöse Symptomkontrolle, Flüssigkeitssubstitution in der Terminalphase, interventionelle Therapie und Physiotherapie in der Palliativmedizin)

2. psychische, soziale und spirituelle Aspekte (Berücksichtigung der psychischen, sozialen und spirituellen Bedürfnisse des Patienten, der Angehörigen und des Behandlungsteams sowohl bei Krankheit, beim Sterben und in der Zeit danach)

3. ethische und rechtliche Fragen (intensive Auseinandersetzung mit speziellen Fragen der Kommunikation, der Ethik, der Arzt-Patientenbeziehung, Selbstbestimmung des Patienten, Grenzen der Behandlung, Sterbehilfe und Sterbebeistand)

Die im Curriculum Palliativmedizin aufgeführten Inhalte und Ziele können unter verschiedenen organisatorischen Rahmenbedingungen sowohl im ambulanten wie im stationären Bereich verfolgt werden. Der stationäre Bereich (Palliativstationen und Hospize) ermöglicht die Betreuung und Behandlung von Patienten, die durch den ambulanten Bereich (ambulanter Palliativdienst, ambulanter Hospizdienst, niedergelassene Ärzte mit palliativmedizinischer Weiterbildung) nicht mehr ausreichend versorgt werden können. Ziel der stationären Einrichtungen sollte es, falls erwünscht, sein, dem Patienten das Sterben zu Hause in seiner vertrauten Umgebung bei seinen Angehörigen und Freunden $\mathrm{zu}$ ermöglichen. Im Rahmen der palliativmedizinischen Betreuung werden physische, psychische, soziale, spirituelle sowie ethisch-rechtliche Aspekte berücksichtigt.

\section{Palliativpatient:}

„Palliativpatienten“ haben eine begrenzte Lebenserwartung und leiden an den Symptomen einer inkurablen, progredienten und weit fortgeschrittenen Erkrankung. Dies ist die Beschreibung der Deutschen Gesellschaft für Palliativmedizin (2003) und der European Association for Palliative Care (2010) in Anlehnung an die Definition der Weltgesundheitsorganisation (1990). Die Mehrzahl der Palliativpatienten ist tumorkrank, aber auch Patienten mit unheilbaren neurologischen und chronisch internistischen Erkrankungen werden palliativmedizinisch betreut. 


\subsection{Therapieziele und Diagnostik in der Palliativmedizin}

Unter der Palliativtherapie versteht man die antineoplastische Therapie, die bei fehlendem kurativem Ansatz Einfluss auf die Tumorerkrankung selbst nimmt (Klaschik, 2001). Genauso wie die Übergänge von kurativer zu palliativer Therapie oft fließend sind, befinden sich die Patienten in einem dynamischen Prozess und durchlaufen mehrere Phasen, die im Rahmen der Behandlungsoptionen berücksichtigt werden müssen. Wichtig ist dabei, sich im Therapieverlauf das unmittelbare Therapieziel immer wieder bewusst $\mathrm{zu}$ machen, dieses $\mathrm{zu}$ hinterfragen und einen eventuellen Therapieabbruch, wenn es die Situation des Patienten erfordern sollte, zu akzeptieren (Samonigg et al., 2000). Eine gebräuchliche Einteilung der Lebensphasen palliativmedizinischer Patienten, die durch die Kölner Palliativmedizinerin Dr. JonenThielemann (2007) formuliert wurde, unterscheidet Rehabilitations-, Präterminal-, Terminal- und Sterbephase. Die Einordnung erfolgt klinisch nach erwarteter Prognose und Aktivität. Der Schwerpunkt palliativmedizinischer Behandlung liegt in der Rehabilitationsphase. In dieser Phase der letzten Monate - selten Jahre - des Lebens wird ein trotz Fortschreiten der Erkrankung weitgehend normales, aktives Leben angestrebt (Klaschik, 2001). Häufige Symptome von Palliativpatienten sind: Schmerzen, Luftnot, Übelkeit, Erbrechen, Husten, Obstipation, intestinale Obstruktion, Verwirrtheit, Geruchsbildung oder präfinale Rasselatmung (Conill et al., 1997; Grond et al., 1994; Aulbert, 1998; Radbruch et al., 2003). Bei der symptomorientierten medikamentösen Therapie kommt der ethische Konflikt zwischen einer Verzögerung des Sterbeprozesses mit möglicher Verlängerung von Leid und einer möglichen Lebensverlängerung mit guter Lebensqualität zum Tragen. Hier sollte im Team und mit dem Patienten und seinen Angehörigen ein Konsens gefunden werde, der die Wünsche und Bedürfnisse des Patienten respektiert (Aulbert et al., 2008).

$\mathrm{Zu}$ dem grundsätzlichen Einsatz von Diagnostik findet man in der Literatur keine konkreten Aussagen und Untersuchungen. Es scheint, als ob die Tatsache, dass die Palliativmedizin ein multidisziplinäres Querschnittsfach mit unterschiedlichen Fachrichtungen ist, kaum allgemeine Aussagen zu diagnostischen Standards zulässt. Es existieren zwar zahlreiche Leitlinien zu speziellen Teilgebieten der Palliativmedizin und in der palliativen Onkologie: medikamentöse Schmerztherapie, Prinzipien der medikamentösen antineoplastischen Systemtherapie, Leitlinien der Radionuklidtherapie bei schmerzhaften Knochenmetastasen, Prinzipien der modernen 
Strahlentherapie usw., für die Gesamtheit palliativmedizinischer Behandlungen und den Einsatz von Diagnostik gibt es aber bisher keine umfassenden Leit- oder Richtlinien (Becker-Schwarze, 2007).

\subsection{Hospiz- und Palliativerhebung (HOPE) - Patientenregister und Forschungsinstrument}

Die 1996 vom Bundesgesundheitsministerium initiierte Arbeitsgemeinschaft mit dem Auftrag der Entwicklung eines Dokumentationssystems zur standardisierten Erfassung von personen-, krankheits- und therapiebezogenen Daten zum Aufnahmezeitpunkt und Behandlungsende von Palliativpatienten in den verschiedenen Einrichtungen entstand in Zusammenarbeit mit der Deutschen Gesellschaft für Palliativmedizin (DGP) und der Deutschen Krebsgesellschaft (DKG). In Kooperation kam 2002 der heutige Deutsche Hospiz- und PalliativVerband (DHPV, damals Bundesarbeitsgemeinschaft Hospiz (BAG Hospiz)) hinzu. Die Hospiz- und Palliativ-Erhebung (HOPE) wird seit 1999 im jährlichen Rhythmus durch eine große Anzahl von Einrichtungen angewendet, so dass die Datenbank von HOPE mittlerweile auf einen großen Datenpool mit 16983 Patientendatensätzen zurückgreifen kann (vgl. Anhang 7.3), mit dessen Hilfe repräsentative Auswertungen zur Palliativversorgung in Deutschland möglich sind.

Die große Zahl der beteiligten Einrichtungen, die Tatsache, dass sich HOPE auf die klinische Praxis bezieht und die regelmäßige Anpassung mit Erweiterung des Systems führen dazu, dass HOPE mittlerweile als Qualitätssicherungsinstrument mit Benchmarkingfunktion eingesetzt wird (Radbruch et al., 2002; Radbruch und Nauck, 2008). Der zweiseitige Basisbogen wird als Standarddokumentation für Palliativpatienten von der Deutschen Gesellschaft für Palliativmedizin empfohlen (Bausewein et al., 2007). Die erhobenen Daten geben Aufschluss über die betreuten Patienten, Therapiemaßnahmen und -erfolge und ermöglichen einen Vergleich zwischen den teilnehmenden Einrichtungen. Die Einführung von weiteren Modulen auf der Basis von spezifischen Forschungsfragen erweitert das Patientenregister $\mathrm{zu}$ einer Forschungsplattform. Finanziert wird HOPE durch die DKG, die DGP, den Deutschen Hospiz- und PalliativVerband und die Firma Mundipharma Limburg. Für die Teilnahme an HOPE gibt es keine speziellen Voraussetzungen. Es ist lediglich die Ernennung einer 
Kontaktperson, die an einem Vorbereitungstreffen teilnimmt und die die Datenerhebung in ihrer Einrichtung koordiniert, erforderlich. Die Anmeldung kann wahlweise zur Online-Dokumentation oder zur handschriftlichen Dokumentation erfolgen. Informationen und Anmeldeformulare sind auf folgenden Homepages: www.dgpalliativmedizin.de, www.palliativmedizin.ukaachen.de und www.hope-clara.de abrufbar oder schriftlich über die dort angegebenen Adressen anzufordern. Im Jahre 2009 wurde erstmalig für die Einrichtungen, die eine Papierdokumentation wünschten, eine Schutzgebühr von €65 für die Basisbögen und €10 für jedes weitere Modul (für Druck und Dateneingabe für bis zu 30 Patienten) erhoben. Die Online-Dokumentation in der internetbasierten elektronischen Datenbank CLARA (Clinical Analysis, Research and Application, auf www.hope-clara.de) blieb kostenfrei (Deutsche Gsellschaft für Palliativmedizin, 2009). Vor Studienbeginn werden die Patienten von Ärzten und/oder dem Pflegepersonal über die Studie informiert und geben schriftlich ihre Zustimmung zur Erhebung und Speicherung der Daten (vgl. Anhang 7.2).

Das modulare System von HOPE besteht aus der sogenannten Kerndokumentation (Basisbogen), der vom Pflegepersonal und/oder dem Arzt, von Psychologen, Sozialarbeitern oder Ehrenamtlichen aus Sicht des Betreuerteams ausgefüllt wird. Es werden demographische und erkrankungsspezifische Daten (Erstdiagnose, Metastasierung bei Tumorerkrankungen, relevante Begleiterkrankungen, körperliche Symptome, pflegerische und psychosoziale Daten) erfasst (vgl. Anhang 7.1.1). Des Weiteren existieren verschiedene Fragebögen/Module mit spezifischen Fragestellungen zu verschiedenen wissenschaftlichen Themen (vgl. 3. Methodik). So wurde 2007 unter anderem erstmals das von der Untersucherin entwickelte Modul zur erweiterten Diagnostik (DIA) eingesetzt und ausgewertet, indem der Einsatz von spezifischen Verfahren (mikrobiologische und bildgebende Verfahren) und deren Folgen und Konsequenzen für Palliativpatienten abgefragt wurden, das unter anderem Grundlage für diese wissenschaftliche Arbeit ist.

HOPE wurde bereits in der Vergangenheit $\mathrm{zu}$ wissenschaftlichen Fragestellungen herangezogen (Bausewein et al., 2005; Radbruch et al., 2000) und ist damit wesentliche Grundlage für Forschung in der Palliativmedizin in Deutschland. 


\subsection{Sonographie}

Die Sonographie ist deutschlandweit das am häufigsten eingesetzte nichtinvasive bildgebende Verfahren und gängiger Bestandteil der erweiterten klinischen Untersuchung (Kassenärztliche Bundesvereinigung, 2009). Sie ist problemlos verfügbar, basiert auf dem Einsatz nichtionisierender Strahlen und unterliegt im Gegensatz zu anderen Schnittbildverfahren keinen patientenbezogenen Kontraindikationen. Zudem erlaubt die kontinuierliche Bildgebung in Echtzeit die Beantwortung morphologischer und funktioneller Fragestellungen (Mariani und Setlaj, 2010). Es dient zur Statuserhebung und hilft bei der Beantwortung vieler differentialdiagnostischer Fragen. Es findet seinen Einsatz in nahezu jedem medizinischen Fachgebiet. Da die Palliativmedizin ein multidisziplinäres Fach ist und mit vielseitigen klinischen Fragestellungen konfrontiert wird, ist es auch hier ein ideales bildgebendes Verfahren. Fragestellungen an die sonomorphologische Bildgebung in der Palliativmedizin zielen u.a. auf das Erkennen von tumor- oder therapieassoziierten Komplikationen, die Abklärung eventuell spezifisch behandelbarer Symptome wie Schmerzen, Luftnot, Erbrechen, die prognostische Neueinschätzung im Hinblick auf Tumorwachstum und Dynamik ab. Zu diesen Fragestellungen gibt es in der Literatur einige Studien, die den Einsatz von Ultraschall, zum Zweck der Diagnostik empfehlen. Mit dem Einsatz moderner Ultraschalldiagnostik können Komplikationen früher erkannt und behandelt werden. Ebenfalls ermöglicht die Sonographie ein schnelles Staging und eine Abschätzung und Einschätzung der aktuellen gesundheitlichen Situation des Patienten. Die Tatsache der Unschädlichkeit der eingesetzten Schallwellen und die leichte und fast flächendeckende Verfügbarkeit ist ein weiterer Vorteil für den Einsatz in der Palliativmedizin. Zudem können einige wichtige Befunde wie z.B. Harnstau oder das Erkennen freier Flüssigkeit auch rasch vom nichtgeübten Untersucher erlernt und angewendet werden (Gebel, 2000). Die Studie „Bedside ultrasound- experience in palliative care units" (Gishen und Trotman, 2009) geht sogar davon aus, dass ein stationseigenes Ultraschallgerät auf Palliativstationen zu einer Liegezeitverkürzung führt, weil unnötige Wartezeiten in der für die Sonographie zuständigen Abteilung und Wege dorthin vermieden werden können (Gishen und Trotman, 2009). Einen weiteren Vorteil sehen Gishen und Trotman in der Flexibilität und Vielseitigkeit des Einsatzes. Zwar führte Gishen die meisten Untersuchungen (52,5\%) zum Nachweis eines Aszites und einer anschließenden sonographisch gesteuerten Punktion durch, er schreibt aber 
auch, dass die Sonographie bei der Darstellung und Beurteilung der harnableitenden Organe, der Pleura, der abdominellen Metastasensuche, bei Blasenfunktionsstörungen oder bei Fragestellungen bezüglich tiefer Beinvenenthrombosen sinnvoll ist. Einen weiteren ganz anderen Benefit sehen die Autoren in der Einsparung der Kosten. Gishen zufolge kostet eine sonographische Untersuchung in einem radiologischen Zentrum ca. $€$ 67 (Department of Health, 2007). Diese Kosten schließen aber nicht weitere Betreuungs- oder Transportkosten ein. Im Vergleich dazu betrugen die Kosten pro Untersuchung in Gishens Studie ca. $£$ 20. Aus Gishens Studie ziehen die Autoren (Gishen und Trotman, 2009) das Fazit, dass der Einsatz von modernen Untersuchungstechniken in der Palliativmedizin durchaus ratsam ist und gefördert werden sollte, da dies ihrer Meinung nach zu einer Verbesserung der Behandlung führt („We belive that by adopting a proactive technical approach the use of this technique can enhance the quality of care we give our patients." (Gishen und Trotman, 2009, S.43)). Leider gibt es zu dem allgemeinen Einsatz der Sonographie in der Forschung kaum weitere wissenschaftliche Studien. Dieser Umstand führte zu der Fragestellung, welche Bedeutung sonographische Untersuchungen in der Palliativmedizin haben und was für Konsequenzen sich daraus ergeben. 


\section{Fragestellung}

Aus den oben dargestellten wissenschaftlichen Grundlagen ergibt sich die Frage, ob und mit welcher Indikation nichtinvasive Diagnostik in dem palliativmedizinischen Setting zum Einsatz kommt. Zu diskutieren ist ebenfalls die Problematik der Belastung für die Patienten und ob sich diese evtl. minimieren ließe. Die vorliegende Untersuchung soll erstmalig dokumentieren, in welchem Ausmaß und mit welcher klinischtherapeutischen Relevanz nichtinvasive Diagnostik im palliativmedizinischen Alltag eingesetzt wird. Dies erfolgt am Beispiel der mikrobiologischen Labordiagnostik und der Bildgebung (Sonographie, konventionelles Röntgen, Schnittbildgebung (CT/MRT) und nuklearmedizinische Diagnostik).

Ein weiterer Schwerpunkt dieser Arbeit ist die Auswertung der 70 sonographischen Untersuchungen, die auf der Palliativstation am Universitätsklinikum Göttingen (Abteilung Palliativmedizin, Zentrum Anaesthesiologie, Rettungs- und Intensivmedizin) durchgeführt und dokumentiert wurden. Diese sollen beispielhaft für nichtinvasive diagnostische Maßnahmen - unter den genannten Aspekten der therapeutischen Relevanz und der Belastung für den Palliativpatienten - diskutiert werden.

Im Einzelnen sollen, anhand der Ergebnisse der HOPE-Studie und der abteilungsinternen Sonographien insbesondere folgende Fragen beantwortet werden:

- In welchem Ausmaß findet in der stationären Palliativmedizin nichtinvasive Diagnostik statt? Wie viele Patienten erhielten nichtinvasive Diagnostik? Welchen Stellenwert hat die Sonographie in der Palliativmedizin?

- Gibt es einen Unterschied in der Einsatzhäufigkeit zwischen Palliativstationen und onkologischen Stationen?

- Nach welchen Untersuchungen (bildgebende-/ mikrobiologische Verfahren) kam es wie häufig und in welchem klinischen Ausmaß zu welchen therapeutischen Konsequenzen?

- Welche Probleme traten bei der Durchführung von diagnostischen Maßnahmen auf? Wie belastend ist die nichtinvasive Diagnostik für den Patienten?

- Gibt es Möglichleiten, die Belastungen, die durch die Durchführung für den Patienten entstehen, zu minimieren? 
- Wie häufig kam es zu Problemen bei der Beurteilbarkeit des mikrobiologischen Laborergebnisses bzw. der Bildgebung?

Durch die Beantwortung dieser Fragen soll ein Zustandsbericht des bisherigen Einsatzes nichtinvasiver Diagnostik in stationären palliativmedizinischen Versorgungsbereichen gegeben werden, um daraus Möglichkeiten zur Verbesserung der Behandlung von Palliativpatienten abzuleiten. Dabei werden mit Hilfe einer quantitativen Datenerfassung erstmalig der besondere Aspekt der Sonographie als alleiniges bildgebendes Verfahren und deren Relevanz und Konsequenzen für die Patienten im palliativmedizinischen Setting betrachtet. 


\section{Methodik}

Die vorliegende Untersuchung zur Beantwortung der oben gestellten Fragen basiert auf zwei Datenquellen: die der Hospiz- und Palliativerhebung HOPE und die der abteilungsinternen Dokumentation.

\subsection{Hospiz- und Palliativerhebung (HOPE)}

Um die in der Einleitung und Abschnitt 2 gestellten Fragen zu beantworten, wurde von der Untersucherin das Modul Diagnostik (DIA) der Hospiz- und Palliativerhebung, in dem Aspekte zur Qualität und Quantität von bildgebenden und mikrobiologischen Verfahren abgefragt wurden, entwickelt und ausgewertet. Weiterhin wurden die Teilergebnisse des Basisbogens (sog. Kerndokumentation) der HOPE-Dokumentation im Erhebungszeitraum von März bis Juni 2007 ausgewertet, um weitere demographische Fakten und spezielle Informationen der Patienten zu nutzen. Zusätzlich wurden 70 Ultraschalluntersuchungen auf der Palliativstation der Universitätsmedizin Göttingen (UMG) erfasst und ausgewertet.

Die Hospiz- und Palliativerhebung HOPE beinhaltet die Erfassung grundlegender Daten palliativmedizinisch oder in einem Hospiz behandelter und begleiteter Patienten in stationären oder ambulanten Versorgungseinrichtungen. HOPE wird durch die Deutsche Gesellschaft für Palliativmedizin (DGP), die Deutsche Krebsgesellschaft (DKG) und die Firma Mundipharma Limburg gefördert und arbeitet seit 1999 mit Palliativstationen, onkologischen Stationen, stationären Hospizen und ambulant tätigen Ärzten zusammen, die zur Teilnahme an der Dokumentation eingeladen werden.

Bei HOPE werden über den Zeitraum vom 15.03. - 15.06. eines jeden Jahres Daten von maximal 30 Patienten einer teilnehmenden Einrichtung nach Einholung einer schriftlichen Einverständniserklärung pseudonymisiert erfasst. Eine Dokumentation der Daten erfolgt überwiegend online mit einer zu diesem Zweck eingerichteten Datenbank (www.hope-clara.de) oder handschriftlich auf den angeforderten und ausgedruckten Dokumentationsbögen. Der über ein Kennwort und einen Zugangscode geschützte Online-Zugang war über den gesamten Dokumentationszeitraum eingerichtet, die Daten konnten von der eingebenden Einrichtung eingesehen und ggf. korrigiert werden. Die Dokumentationsbögen sollten bis zum 30.6.2007 online in die Datenbank eingetragen 
oder auf Papier ausgefüllt und per Post zur Auswertung versandt werden. Dokumentationsbögen, die nach dem 30.06.2007 eintrafen, wurden nicht mehr in die Auswertung einbezogen. Patienten, die während der Dokumentationsphase entlassen worden sind, durften bei einer Wiedervorstellung nicht erneut in die Dokumentation aufgenommen werden.

Zur Dokumentation standen folgende Bögen zur Verfügung:

- Basisbogen: Es sollten mindestens zwei Basisbögen pro Patient ausgefüllt werden, einer zu Beginn (Aufnahmebogen) und einer am Ende (Abschlussbogen) (vgl. Anhang 7.1). Mit Hilfe dieser Dokumentationsbögen wurden grundlegende Eckdaten der medizinischen Krankheitsvorgeschichte, der aktuellen klinischen, pflegerischen und psychosozialen Problematik und der stattgehabten therapeutischen Interventionen $\mathrm{zu}$ Beginn, im Verlauf und am Ende einer (stationären oder ambulanten) Behandlung (Entlassung bzw. Versterben) dokumentiert.

Des Weiteren konnten von den teilnehmenden Einrichtungen optional verschiedene Modulbögen angefordert und ausgefüllt werden:

- DIA: Erfassung von diagnostischen Maßnahmen

In dem speziell für diese Fragestellung von der Untersucherin neu entwickelten Modul wurden beispielhaft für diagnostische Maßnahmen in der Palliativmedizin die Daten von Patienten mit mikrobiologischen und bildgebenden Verfahren (Sonographie, Röntgen, CT/MRT und nuklearmedizinischer Diagnostik) dokumentiert (vgl. Anhang 7.1). Die ausfüllende Person konnte in dem Modul DIA nicht nur Angaben zu der Qualität und Quantität der durchgeführten Untersuchungen machen, sondern auch über die therapeutische Relevanz und die Probleme, die während und durch die Untersuchung für den Patienten entstanden sind. Der Bogen sollte einmalig retrospektiv bei der Entlassung des Patienten ausgefüllt werden.

Darüber hinaus konnten folgende Module konsekutiv von den teilnehmenden Einrichtungen ausgefüllt werden: 
- MIDOS: die Selbsterfassung von Schmerzen, weiteren Symptomen und Allgemeinbefinden

- LCP: die Analyse der palliativen Betreuung am Lebensende nach dem Liverpool Care Pathway oft he Dying

- TT: die Erfassung von tumorspezifischen Therapien.

Weitere Module waren in vorangegangenen Jahren in Ergänzung zum Basisbogen genutzt worden:

- ein persönlicher Bogen zur Dokumentation wichtiger Adressen für die Betreuung

- der Palliative Outcome Scale (POS) in den Versionen für die Selbst- und Fremderfassung

- die ehrenamtliche Begleitung

- die Trauerbegleitung

- die kontinuierliche Symptomerfassung (Wochenbogen)

- ein Therapieplan zur Erfassung der therapeutischen Maßnahmen

- die Erfassung der Selbstständigkeit bzw. des Hilfe- und Pflegebedarfs

- ein Bogen zur Dokumentation des Behandlungsaufwandes (für stationäre Einrichtungen)

- die ethischen Konflikte und Probleme - Entscheidungen

- die Einschätzung der Prognose

- der psychoonkologische Basisdokumentationsbogen (PoBaDo).

Das Modul DIA bestand aus einem Dokumentationsbogen mit Ankreuzmöglichkeit. Teilweise war eine Einfach-, in manchen Punkten war eine Mehrfachauswahl möglich. Die Einrichtungen hatten die Möglichkeit, die Daten des Bogens online selbst einzugeben (http://www.hope-clara.de/) oder handschriftlich auszufüllen und an das Institut CLARA (Clinical Analysis, Research and Application) zurückzusenden. Da das DIA-Modul ein Zusatzmodul zu dem bereits vorhandenen anonymisierten Basisbogen darstellte, war eine erneute Anonymisierung dieser Daten nicht notwendig. 
Die bereits in elektronischer Form vorliegenden Datensätze wurden in anonymisierter Form durch das Institut CLARA zur Auswertung an die Universitätsmedizin Göttingen weiter gereicht. Der nach Beendigung des Erhebungszeitraums noch nicht elektronisierte Teil der Daten wurde von Hand eingegeben, elektronisch gespeichert (MS-Excel 2008) und ausgewertet.

Das DIA-Modul erfasste die mikrobiologischen Untersuchungen und die daraus entstehenden therapeutischen Konsequenzen (Teil I; DI1(a.-b.)-DI2.) getrennt von den bildgebenden Verfahren und deren therapeutischen Konsequenzen (Teil II; DI3(a.-b.)DI8). Beide Verfahren mussten hinsichtlich ihres technisch/analytischen Ergebnisses bewertet werden. Hier war entweder „Ja“ oder „Nein“ anzukreuzen. Bei der Auswertung des DIA-Moduls wurden bei der prozentualen Berechnung im Hinblick auf ihr „technisch/analytisches Ergebnis“ nur die Bögen, bei denen eindeutig „Ja“ angekreuzt wurde, als eindeutig positiv und alle, die mit „Nein“ beantwortetet waren, als eindeutig negativ berücksichtigt.

Probleme bei der Beurteilbarkeit (DI7b.) und Durchführung (DI7a.) konnten nur bei der Bildgebung angegeben werden. Im letzten Abschnitt des Fragebogens (DI9.) wurde dokumentiert, von wem der Bogen ausgefüllt worden war (Berufsgruppe).

\subsection{Abteilungsinterne Dokumentation}

Im Rahmen der prospektiven Studie auf der Palliativstation des Universitätsklinikums in Göttingen (UMG) wurden über einen Zeitraum von 10 Monaten (Oktober 2006 bis August 2007) konsekutiv 70 Sonographien dokumentiert. In diesem Untersuchungsteil wurden die Resultate der 70 Ultraschalluntersuchungen mit Hilfe der abteilungsinternen Standarddokumentation der Abteilung Palliativmedizin zur Auswertung herangezogen. Hierfür wurden alle Patienten der Abteilung Palliativmedizin, die in dem o.g. Zeitraum eine sonographische Untersuchung erfuhren, in Bezug auf anamnestische Angaben, klinische und technische Befunde und spezielle Fragestellung erfasst. Eine Selektion nach Fragestellung oder Krankheitsphase hat nicht stattgefunden, damit ein möglichst breitgefächertes und vielseitiges Patientenkollektiv dokumentiert werden konnte. Die Ultraschalluntersuchungen wurden von dem behandelnden Arzt in der Abteilung Palliativmedizin als unabhängige Person 
durchgeführt. Da die Abteilung über ein stationseigenes Ultraschallgerät (Philips HD3, Schallkopf: 3,5 Megahertz, curved array) verfügt, konnten die Ultraschalluntersuchungen problemlos zu jedem Zeitpunkt durchgeführt werden.

Die Untersuchungen erfolgten inhaltlich und personell unabhängig von der oben genannten HOPE-Dokumentation. Zum Zweck der besseren Vergleichbarkeit wurde von der Untersucherin ein weiterer Dokumentationsbogen entwickelt, der sich an dem DIAModul der HOPE-Dokumentation orientiert (vgl. Anhang 7.4) Anschließend wurden die gewonnenen Daten mit Hilfe von Microsoft Excel (2008) für Mac elektronisch gespeichert. Exemplarisch für nichtinvasive diagnostische Maßnahmen wurden die Ergebnisse nach Aspekten der therapeutischen Relevanz, der Konsequenzen und der Belastung / Probleme für den Patienten ausgewertet.

Die Patienten wurden durch Vergabe von Nummern pseudonymisiert und mit dem Programm „Excel“ zwischenarchiviert. Es wurden Basisdaten wie Alter, Geschlecht und Verweildauer dokumentiert. In Stichpunkten wurde die Indikation der Untersuchung festgehalten.

Das Ergebnis der Untersuchung und die daraus resultierenden Konsequenzen konnten nach folgenden Punkten sortiert werden:

- Symptomabklärung / Verlaufskontrolle ohne weitere Konsequenzen im Bezug auf die aktuelle Therapie

- nachfolgendes invasives Verfahren (Punktion, Endoskopie, Blasenkatheter, etc.)

- nachfolgendes nicht invasives Verfahren (Abführmaßnahmen, Ileustherapie, Antibiose, Diuretika, etc.)

- prognostische Neubewertung mit Abbruch der aktuellen Therapie

- prognostische Neubewertung mit Therapiezieländerung oder neuer Therapie

- Konsiliarische Untersuchung.

Zusätzlich musste das Ergebnis der Untersuchung hinsichtlich ihres technischanalytischen Ergebnisses bewertet werden. Am Ende konnte angegeben werden, ob Probleme bei der Beurteilbarkeit und / oder Durchführung aufgetreten waren. 


\subsection{Allgemeine Anmerkungen zur Methodik und Statistik}

Die Auswertung der Daten erfolgte deskriptiv. In den Bereichen Bildgebung und Mikrobiologie erfolgte ein Vergleich zwischen Palliativstationen und onkologischen Stationen. Auch bei einigen demographischen Fakten und Ergebnissen der Dokumentation des Basisbogens wurden die beiden Einrichtungen miteinander verglichen. Die Übertragung der Rohdaten von der online Datenbank in das Kalkulationsprogramm Microsoft Office Excel (2003) für Windows sowie eine erste statistische Auswertung mit Hilfe des Statistik-Programm-Systems für Sozialwissenschaften (SPSS), Version 8.0 für Windows, erfolgte durch das Daten verarbeitende Institut CLARA sowie durch die Autorin.

Die weitere deskriptive und analytische Auswertung der Daten, die Berechnung von Mittelwerten und von prozentualen Anteilen erfolgte mit Hilfe von Microsoft Excel (2008) für Mac. Signifikanzberechnungen wurden mit STATISTICA durchgeführt. Hierbei wurde zur Signifikanzberechnung der Chi-Quadrat-Vierfeldertest und der Wilcoxon-Rangsummentest eingesetzt. Die statistische Signifikanz wurde auf dem Niveau $\alpha=5 \%$ festgelegt.

Statistikberatungstermine erfolgten im Zentrum für Statistik der Universität Göttingen am 16.09.2008 und am 22.09.2008.

Die Studie wurde von der Ethikkommission der Medizinischen Fakultät Göttingen in der Sitzung vom 20.09.2007 zustimmend bewertet. 


\section{Ergebnisse}

\subsection{Ergebnisse HOPE}

\subsubsection{Teilnehmende Einrichtungen an HOPE und DIA-Modul-Rücklauf der HOPE - Dokumentation}

An HOPE nahmen im Erhebungszeitraum 2007 insgesamt 149 Einrichtungen (3184 Patienten) teil. Die Einrichtungen verteilten sich wie folgt:

\begin{tabular}{|c|c|c|c|}
\hline & Anzahl der & Anzahl der & $\begin{array}{c}\text { Anzahl der } \\
\text { Patienten mit DIA- }\end{array}$ \\
\hline Palliativstation & 67 & 1663 & 565 \\
\hline Onkologische Station & 8 & 143 & 30 \\
\hline Hospiz & 24 & 498 & 0 \\
\hline Ambulant tätiger Arzt & 28 & 435 & 60 \\
\hline Ambulanter Pflegedienst & 22 & 445 & 36 \\
\hline
\end{tabular}

Tabelle 2: Auflistung teilnehmender Einrichtungen

Im Durchschnitt aller Einrichtungen wurden 21,4 Therapieverläufe erfasst.

Insgesamt nahmen 99 stationäre Einrichtungen (67 Palliativstationen, 8 onkologische Stationen, 24 Hospize) und 50 ambulante Einrichtungen (28 ambulant tätige Ärzte und 22 ambulante Pflegedienste) an HOPE teil. Dabei wurden die Krankheitsverläufe von 2304 stationären Patienten (1663 Patienten von Palliativstationen und 143 Patienten von onkologischen Stationen, 498 Patienten aus dem Hospiz) und 880 ambulanten Patienten dokumentiert. Für 595 von 2304 stationären Patienten wurde ein DIA-Modul erstellt, von den 880 ambulanten Patienten wurde für 96 Patienten ein DIA-Modul erstellt. Somit wurde das Modul Diagnostik (DIA) insgesamt 691mal ausgefüllt (vgl. Tabelle 2).

Da 86,1\% (595) aller DIA-Bögen von stationären Einrichtungen ausgefüllt wurden (Palliativstation 565, onkologische Station 30, Hospiz 0), beziehe ich mich bei der 
weiteren Beschreibung und der Diskussion der Resultate im Sinne der besseren Vergleichbarkeit ausschließlich auf die beiden stationären Versorgungseinrichtungen Palliativstation und onkologische Station.

\subsubsection{Demographische Daten und Verweildauer des Gesamtkollektivs HOPE}

Das durchschnittliche Alter aller HOPE-Patienten auf Palliativstationen betrug 67,1 Jahre und die durchschnittliche Verweildauer betrug 13,1d. Die Patienten aller teilnehmenden onkologischen Stationen waren im Durchschnitt 66,3 Jahre alt und hatten eine durchschnittliche stationäre Verweildauer von 13,6d. Die Geschlechterverteilung war ausgewogen. 53,5\% der Patienten der Palliativstationen waren weiblich und 46,5\% männlich. Eine ähnliche Verteilung gab es auch auf onkologischen Stationen, hier waren 50,7\% weiblich und 49,3\% männlich (siehe Tabelle $3)$.

\subsubsection{Demographische Daten und Verweildauer der HOPE-Patienten mit DIA- Modul}

Das durchschnittliche Alter der Patienten mit ausgefülltem DIA-Modul auf Palliativstationen betrug 67,9 Jahre, die durchschnittliche Liegedauer der Patienten belief sich auf 13,1d. Die Palliativpatienten mit DIA-Modul auf onkologischen Stationen hatten ein durchschnittliches Alter von 67,6 Jahren und eine Verweildauer (Mittelwert) von 13,2d. In den beiden Gruppen sind die Verteilungen von Alter (Alter, $p=0,91$ ) und Verweildauer $(p=0,20)$ bei einem Signifikanzniveau von $5 \%$ nicht unterschiedlich lokalisiert. Auch bei den Patienten mit DIA-Modul zeigten sich keine bedeutenden Unterschiede in der Geschlechterverteilung, 55,5\% der Palliativstationspatienten waren weiblich und 44,5\% männlich. Die Geschlechterverteilung der Patienten auf onkologischen Stationen war wieder ähnlich, hier waren 51,9\% weiblich und 48,1\% männlich (siehe Tabelle 3). 


\begin{tabular}{|c|c|c|c|c|}
\hline & \multicolumn{2}{|c|}{ Palliativstationen (PS) } & \multicolumn{2}{|c|}{ Onkologische Station (OS) } \\
\hline & HOPE & DIA & HOPE & DIA \\
\hline$\varnothing$ Alter (Jahre) & 67,2 & 67,9 & 66,3 & 67,6 \\
\hline$\varnothing$ Verweildauer (Tage) & 13,1 & 13,1 & 13,6 & 13,2 \\
\hline Geschlecht weibl. (\%) & 53,5 & 55,5 & 50,7 & 51,9 \\
\hline Geschlecht männl. (\%) & 46,5 & 44,5 & 49,3 & 48,1 \\
\hline
\end{tabular}

Tabelle 3: Gegenüberstellung der demographischen Daten und der Verweildauer der Patienten mit DIA-Modul zum Gesamtkollektiv HOPE

\subsubsection{Auswertung der Rücksendung des DIA-Moduls}

Das Diagnostik (DIA)-Modul wurde 595mal zurückgeschickt, 565mal von Palliativstationen und 30mal von onkologischen Stationen.

Ein leeres nicht ausgefülltes DIA-Modul wurde von 158 Patienten zurückgeschickt, was als Fehlen von bildgebender oder mikrobiologischer Diagnostik interpretiert wurde. Bei den restlichen 437 Patienten wurde entweder ein bildgebendes Verfahren oder eine mikrobiologische Untersuchung dokumentiert. Häufig wurden auch beide Verfahren angewendet, wie in Tabelle 4 dargestellt.

\begin{tabular}{|c|c|c|c|}
\hline & $\begin{array}{l}\text { DIA Palliativstation } \\
\qquad(565)\end{array}$ & $\begin{array}{l}\text { DIA Onkologie } \\
(30)\end{array}$ & $\begin{array}{l}\text { Gesamt } \\
\text { (595) }\end{array}$ \\
\hline keine Diagnostik & 154 & 4 & 158 \\
\hline nur Bildgebung & 263 & 19 & 282 \\
\hline nur Mikrobiologie & 25 & 1 & 26 \\
\hline $\begin{array}{c}\text { Bildgebung + } \\
\text { Mikrobiologie }\end{array}$ & 123 & 6 & 129 \\
\hline
\end{tabular}

Tabelle 4: Verteilung der einzelnen Verfahren im DIA-Modul 
Eine mikrobiologische Untersuchung als alleiniges diagnostisches Verfahren erfolgte bei 26 Patienten, insgesamt erhielten 155 Patienten mikrobiologische Diagnostik. Bei 282 Patienten wurde ausschließlich bildgebende Diagnostik durchgeführt. Insgesamt kam bei 411 aller Patienten ein bildgebendes diagnostisches Verfahren zur Anwendung.

\subsubsection{Auswertung DIA-Modul Teil I: Mikrobiologische Untersuchungen (DI1a.- DI2)}

Auf den Palliativstationen wurde 148mal eine mikrobiologische Untersuchung durchgeführt, von diesen hatten 142 (89,9\%) eine therapeutische Konsequenz. Bei sieben Patienten auf onkologischen Stationen wurde ebenfalls ein mikrobiologisches Verfahren durchgeführt, hiervon hatten zwei Untersuchungen therapeutische Konsequenzen. Hier ist eine Diskrepanz im Hinblick auf die Einsatzhäufigkeit zwischen den beiden Einrichtungen zu erkennen, da auf Palliativstationen 36\% der erfassten Patienten eine mikrobiologische Untersuchung hatten, auf onkologischen Stationen hingegen $57,1 \%$.

Die Konsequenzen für die Therapie verteilten sich auf folgende Optionen (Tabelle 5):

\begin{tabular}{|c|c|c|c|}
\hline & Palliativstation & Onkologie & Gesamt \\
\hline Verordnung einer Antibiose & $(158)$ & $(5)$ & $(163)$ \\
\hline Verzicht auf eine Antibiose & $111(70,3 \%)$ & $1(20,0 \%)$ & $112(68,7 \%)$ \\
\hline Generelle prognostische Neubewertung & $11(7,0 \%)$ & $0(0,0 \%)$ & $11(6,7 \%)$ \\
\hline Sonstige Konsequenzen & $9(5,7 \%)$ & $0(0,0 \%)$ & $9(5,5 \%)$ \\
\hline Keine Konsequenzen & $11(7,0 \%)$ & $1(20,0 \%)$ & $12(7,3 \%)$ \\
\hline
\end{tabular}

Tabelle 5: Auflistung der therapeutischen Konsequenzen 
Die Tabelle 5 zeigt, dass als häufigste Konsequenz auf Palliativstationen „Verordnung einer Antibiose“ (68,7\%) angegeben wurde. Für den Großteil der Palliativpatienten auf onkologischen Stationen wurden für mikrobiologische Untersuchungen „keine Konsequenzen“ angegeben (60,0\%). Die zur Auswahl stehende Antwort einer „generellen prognostischen Neubewertung" aufgrund des Resultats der mikrobiologischen Untersuchung wurde auf den Palliativstationen nur selten (5,7\%) gewählt, auf onkologischen Stationen sogar nie.

\subsubsection{Auswertung DIA-Modul Teil II: Bildgebende Verfahren (DI3a.-DI8.)}

In den beiden Einrichtungen erhielten insgesamt 411 von den 595 Patienten mindestens ein bildgebendes Verfahren. Im Schnitt wurden bei einem Patienten 1,7 bildgebende Verfahren durchgeführt (684 bildgebende Untersuchungen / 411 Patienten). Hier gab es keinen signifikanten Unterschied zwischen palliativ und onkologischen Stationen (PS 411/ OS 26, $\alpha=0,05, p=0,640$ ) In Bezug auf die verschiedenen diagnostischen Verfahren entfielen auf Palliativstationen 68,3\% (386 abs.) aller Untersuchungen auf ein bildgebendes Verfahren, auf onkologischen Stationen 83,3\% (25 abs.).

Bei der Analyse der Häufigkeit der einzelnen Verfahren zeigte sich, dass in beiden Einrichtungsarten die Sonographie und die konventionelle Röntgendiagnostik die am häufigsten verwendeten Verfahren waren. Auf Palliativstationen erhielt mehr als die Hälfte der Patienten (67,1\%) eine Sonographie oder eine Röntgenuntersuchung $(65,2 \%)$. Bei den onkologisch betreuten Palliativpatienten wurden sogar bei mehr als drei Viertel der Patienten (80,0\%) eine Sonographie und fast $90 \%$ eine Röntgendiagnostik dokumentiert. Ein Schichtbildverfahren (CT/MRT) war das am dritthäufigsten genannte Untersuchungsverfahren (26,6\%), die nuklearmedizinische Untersuchung lag mit 3,1\% an vierter Stelle und war somit das am seltensten angewandte bildgebende Verfahren auf Palliativstationen. Auf onkologischen Stationen lagen die schichtbildgebenden Untersuchungen (CT/MRT) und die nuklearmedizinischen Untersuchungen ebenfalls auf Platz drei und vier. Hier erhielten 44,0\% der Patienten ein CT/MRT und 16,0\% eine nuklearmedizinische Untersuchung. Hier zeigt sich, dass das konventionelle Röntgen und die nuklearmedizinischen Untersuchungen in onkologischen Einrichtungen signifikant häufiger zum Einsatz 
kommen als auf Palliativstationen. Tabelle 6 zeigt die Verteilung der einzelnen Verfahren.

\begin{tabular}{|c|c|c|c|c|}
\hline & DIA PS (386) & DIA OS (25) & Gesamt (411) & $\begin{array}{l}\text { Signifikanzniveau } \\
\alpha=0,05 \text {; p-Werte }\end{array}$ \\
\hline Sonographie & $67,1 \%(259)$ & $80,0 \%(20)$ & $67,9 \%(279)$ & 0,1806 \\
\hline Röntgen & $65,2 \%(252)$ & $88,0 \%(22)$ & $66,7 \%(274)$ & 0,0196 \\
\hline CT/MRT & $26,6 \%(104)$ & $44,0 \%(11)$ & $28,0 \%(115)$ & 0,0656 \\
\hline NUK & $3,1 \%(12)$ & $16,0 \%(4)$ & $3,9 \%(16)$ & 0,0012 \\
\hline
\end{tabular}

Tabelle 6: Prozentuale Verteilung der einzelnen Verfahren auf die Patientenzahl $(n=411)$ bezogen

Aus 81,1\% (313) der bildgebenden Verfahren resultierten auf Palliativstationen therapeutische Konsequenzen für die Patienten, ähnlich wie auf onkologischen Stationen, wo aus $76,0 \%$ der bildgebenden Untersuchungen therapeutische Konsequenzen gezogen wurden. Gesamt gesehen hatten 80,8\% der bildgebenden Verfahren eine Konsequenz für die betroffenen Patienten. Da die bildgebenden Untersuchungen mehr als eine Konsequenz für die Patienten haben konnten (Mehrfachauswahl), wurden insgesamt 491 therapeutische Konsequenzen konkret benannt, 457 auf Palliativstationen und 34 auf onkologischen Stationen.

Die Verteilung der einzelnen Konsequenzen auf die Patienten war, wie Tabelle 7 zeigt, relativ ausgewogen. Auf Palliativstationen resultierte aus bildgebender Diagnostik bei 32,4\% der Patienten ein nachfolgendes nichtinvasives Verfahren (Abführmaßnahmen, Ileustherapie, Diuretika, etc.), in 30,0\% eine prognostische Neubewertung, in 25,6\% eine invasive Maßnahme (Punktion, Endoskopie, Blasenkatheter, etc.). Auf onkologischen Stationen erfuhren nach der bildgebenden Untersuchung 36,0\% der Patienten ein invasives Verfahren und eine prognostische Neubewertung (36,0\%). Am dritthäufigsten resultierte ein nichtinvasives Verfahren (24,0\%). 


\begin{tabular}{|c|c|c|c|}
\hline $\begin{array}{l}\text { Therapeutische } \\
\text { Konsequenzen }\end{array}$ & $\begin{array}{l}\text { DIA Palliativstation } \\
\qquad(386)\end{array}$ & $\begin{array}{l}\text { DIA Onkologie } \\
\text { (25) }\end{array}$ & $\begin{array}{c}\text { Gesamt } \\
(411)\end{array}$ \\
\hline $\begin{array}{c}\text { nachfolgendes } \\
\text { invasives Verfahren }\end{array}$ & $25,6 \%(99)$ & $36,0 \%(9)$ & $26,3 \%(108)$ \\
\hline $\begin{array}{l}\text { nachfolgendes nicht } \\
\text { invasives Verfahren }\end{array}$ & $32,4 \%(125)$ & $24,0 \%(6)$ & $31,9 \%(131)$ \\
\hline $\begin{array}{l}\text { Generelle prognostische } \\
\text { Neubewertung }\end{array}$ & $30,0 \%(108)$ & $36,0 \%(9)$ & $28,5 \%(117)$ \\
\hline Sonstige Konsequenzen & $20,5 \%(79)$ & $4,0 \%(1)$ & $19,5 \%(80)$ \\
\hline Keine Konsequenzen & $11,9 \%(46)$ & $36,0 \%(9)$ & $13,4 \%(55)$ \\
\hline
\end{tabular}

Tabelle 7: Aus bildgebender Diagnostik resultierende therapeutische klinische Konsequenzen

\subsubsection{Auswertung DIA-Modul Teil II: Probleme bei der Durchführung / Beurteilbarkeit}

Im Abschnitt DI7a.-DI7b. konnte die dokumentierende Person Angaben zu Problemen machen, die durch oder bei der Untersuchung für den Patienten entstanden sind. Es konnten sowohl Probleme bei der Durchführung dokumentiert werden, wie zum Beispiel „Schmerzen durch die Untersuchung“ usw. als auch Probleme, die die Beurteilung erschwerten, wie zum Beispiel Meteorismus, Adipositas usw..

Bei der Auswertung zeigte sich hier eine deutliche Diskrepanz zwischen den beiden Einrichtungen. Auf Palliativstationen traten bei 18,4\% der 386 Patienten Probleme bei/durch die Untersuchung auf und bei 6,0\% der Patienten gab es Probleme bei der Beurteilbarkeit. Signifikant $(\alpha=0,05, p<0,001)$ mehr Probleme bei oder durch die Untersuchung gab es auf onkologischen Stationen, hier wurde bei mehr als der Hälfte (56\%) der 25 Patienten Probleme dokumentiert.

Auch in dem Abschnitt „Probleme“ war eine Mehrfachauswahl möglich. Es zeigte sich jedoch, dass in beiden Einrichtungen „Schmerzen durch die Untersuchung“, „Transport und Lagerungsprobleme“ und „Ruhebedürfnis des Patienten“ die drei häufigsten 
Komplikationen waren. Die Abbildungen 1 und 2 zeigen die Verteilung der einzelnen Probleme, Abb.1 stellt die Verteilung auf onkologischen Stationen und Abb.2 die auf Palliativstationen graphisch dar. Auf onkologischen Stationen wurden bei 14 Patienten insgesamt 20 Problemsituationen und auf Palliativstationen bei 71 Patienten insgesamt 99 Problemsituationen dokumentiert.

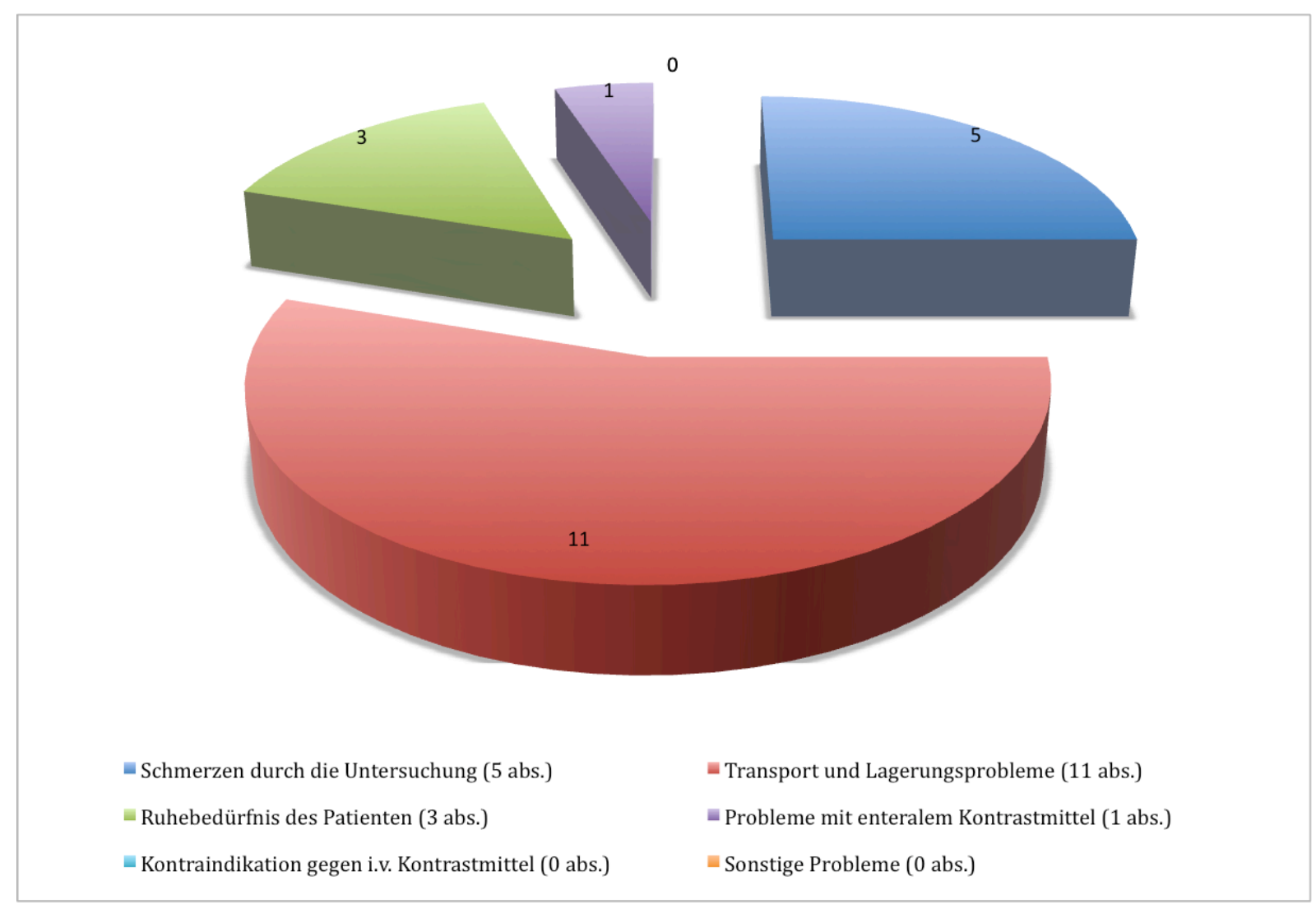

Abbildung 1: Probleme bei der Durchführung OS $(n=20)$ 


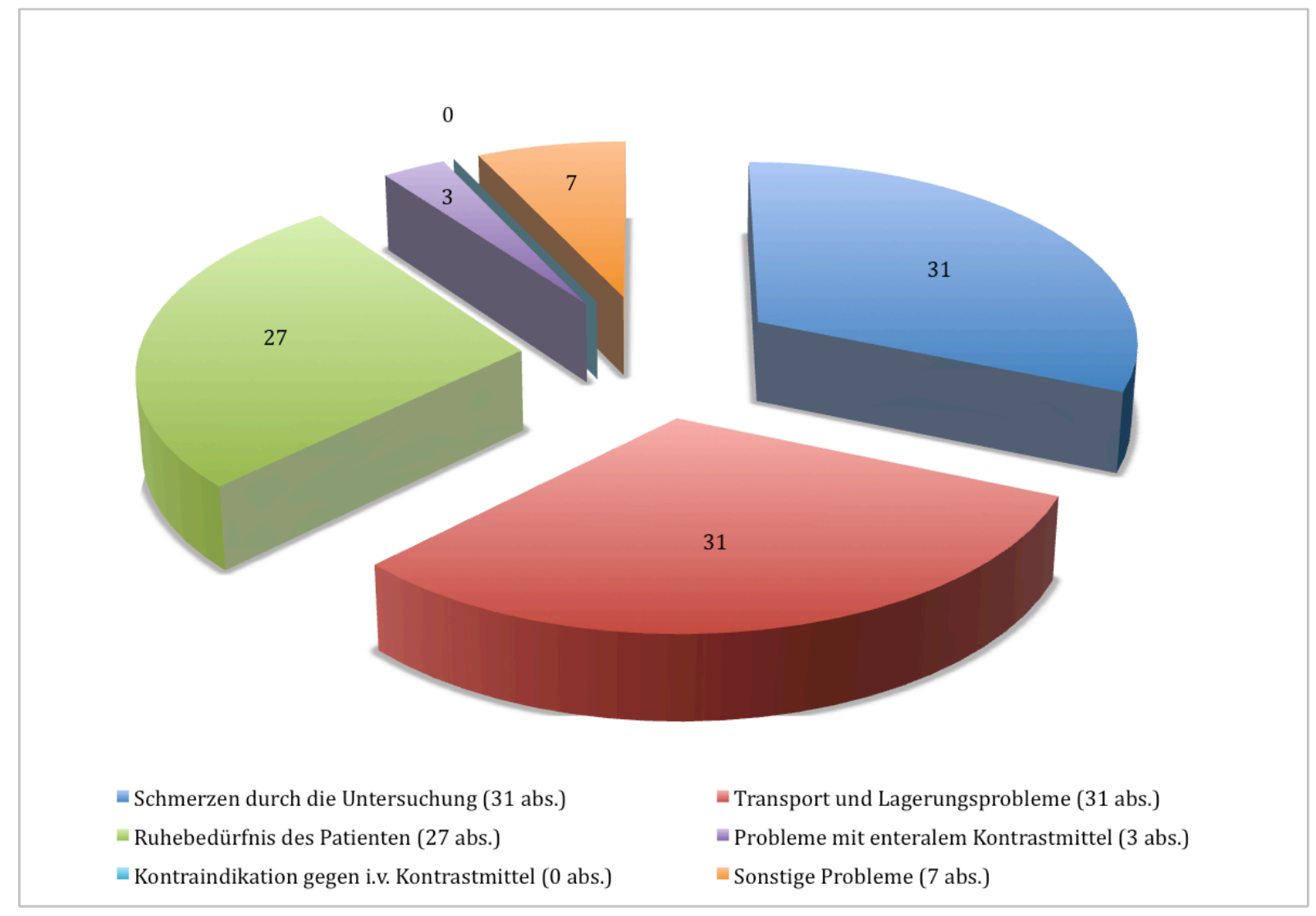

Abbildung 2: Probleme bei der Durchführung PS (n = 99)

\subsubsection{Auswertung DIA-Modul Teil I + II: „technisch-analytisches Ergebnis“}

Die Abschnitte „Mikrobiologische Verfahren“ (Teil I) und „Bildgebende Verfahren“ (Teil II) konnten jeweils am Ende hinsichtlich ihres „technisch-analytischen Ergebnisses“ bewertet werden (Ankreuzoption: Ja / Nein).

Wie Tabelle 8 zeigt, wurden auf Palliativstationen 108 mikrobiologische Untersuchungen als positiv (Ja) und sieben als negativ (Nein) bewertet. In 33 der insgesamt 148 DIA-Module wurde keine Aussage im Bezug auf ihr technisch/analytische Ergebnis gemacht. Somit ist bei der Auswertung der Daten keine eindeutige Zuordnung hinsichtlich ihres technisch/analytischen Ergebnisses möglich, da diese 33 Untersuchungen sowohl positiv als auch negativ ausgefallen sein könnten. Diese Problematik wird im Diskussionsteil weiter erörtert.

Ebenso im zweiten Teil „Bildgebende Verfahren“: Hier wurden 478 als positiv, 10 als negativ und 139 nicht bewertet. 
Ähnliche Ergebnisse zeigten sich bei der Analyse der Daten der onkologischen Stationen (vgl. Tabelle 8). Von den sieben mikrobiologischen Untersuchungen wurden alle eindeutig in Bezug auf ihr technisch-analytisch verwertbares Ergebnis hin bewertet (Ja $=4$; Nein $=3$ ). Bei den 57 bildgebenden Untersuchungen wurden 32 als positiv und drei als negativ bewertet. Bei 22 der bildgebenden Untersuchungen wurden keine Angaben hinsichtlich ihres technisch-analytischen Ergebnisses gemacht.

\begin{tabular}{|c|c|c|c|c|c|c|c|c|}
\hline Technisch- & \multicolumn{2}{|c|}{$\mathrm{Ja}$} & \multicolumn{2}{|c|}{ Nein } & \multicolumn{2}{|c|}{ Keine Angaben } & \multicolumn{2}{|c|}{ Gesamt } \\
\hline Ergebnis & PS & OS & PS & OS & PS & OS & PS & OS \\
\hline $\begin{array}{c}\text { Mikrobiologische } \\
\text { Verfahren }\end{array}$ & 108 & 4 & 7 & 3 & 33 & 0 & 148 & 7 \\
\hline $\begin{array}{c}\text { Bildgebende } \\
\text { Untersuchungen }\end{array}$ & 478 & 32 & 10 & 3 & 139 & 22 & 627 & 57 \\
\hline
\end{tabular}

Tabelle 8: Ergebnisse „technisch-analytisches Ergebnis“; PS = Palliativstationen; OS = onkologische Stationen

\subsection{Datenauswertung der Palliativstation der UMG}

\subsubsection{Demographische Daten und Verweildauer der Patienten der Palliativstation Göttingen}

Auf der Palliativstation in Göttingen wurden in einem Zeitraum von 10 Monaten (10/0608/07) 70 Ultraschalluntersuchungen dokumentiert. Die Patienten hatten eine durchschnittliche Liegedauer von 13,4 Tagen. Das mittlere Alter der Patienten zum Zeitpunkt der Untersuchung lag bei 61,3 Jahren. Mit 38 (54,3\%) weiblichen und 32 $(45,7 \%)$ männlichen Patienten war die Geschlechterverteilung relativ ausgewogen. 


\subsubsection{Auswertung der Sonographieergebnisse der UMG-Patienten}

67 (95,7\%) der 70 Untersuchungen wiesen ein verwertbares Ergebnis auf. Bei drei Patienten musste die Ultraschalluntersuchung aufgrund eines stark ausgeprägten Meteorismus ohne verwertbares Ergebnis beendet werden.

Die Auswertung der Untersuchungsbefunde erfolgte nach demselben Schema wie die Auswertung der HOPE-Daten.

Bei $26(37,2 \%)$ Patienten ergab sich eine therapeutische Konsequenz aus der Untersuchung, bei 44 (62,9\%) Patienten hatte die Untersuchung keine therapeutisch relevanten Konsequenzen. Hier diente die Untersuchung der Symptomabklärung oder der Verlaufskontrolle ohne Auswirkungen auf die aktuelle Therapie. Bei dem Punkt „therapeutische Konsequenzen“ war auch hier eine Mehrfachauswahl möglich. Mit $17,1 \%$ war ein nachfolgendes invasives Verfahren die häufigste Konsequenz. An zweiter Stelle standen die nichtinvasiven Verfahren (12,9\%), wie zum Beispiel Ileustherapie, Antibiose usw..

Bei 5,7\% der Patienten führten die Untersuchungsbefunde zu einer prognostischen Neubewertung mit Abbruch einer tumorspezifischen Therapie. Ebenfalls 5,7\% der Patienten wurden nach der Ultraschalluntersuchung für ein Konsil $\mathrm{zu}$ anderen Fachrichtungen überwiesen. An letzter Stelle stand die „Prognostische Neubewertung mit Änderung / oder neuer Therapie“ (1,4\%).

Somit gab es bei 26 Patienten insgesamt 30 therapeutische Konsequenzen, diese verteilten sich wie folgt (siehe Abb.3): Anteil der einzelnen Verfahren an den therapeutischen Konsequenzen $(n=30)$. 


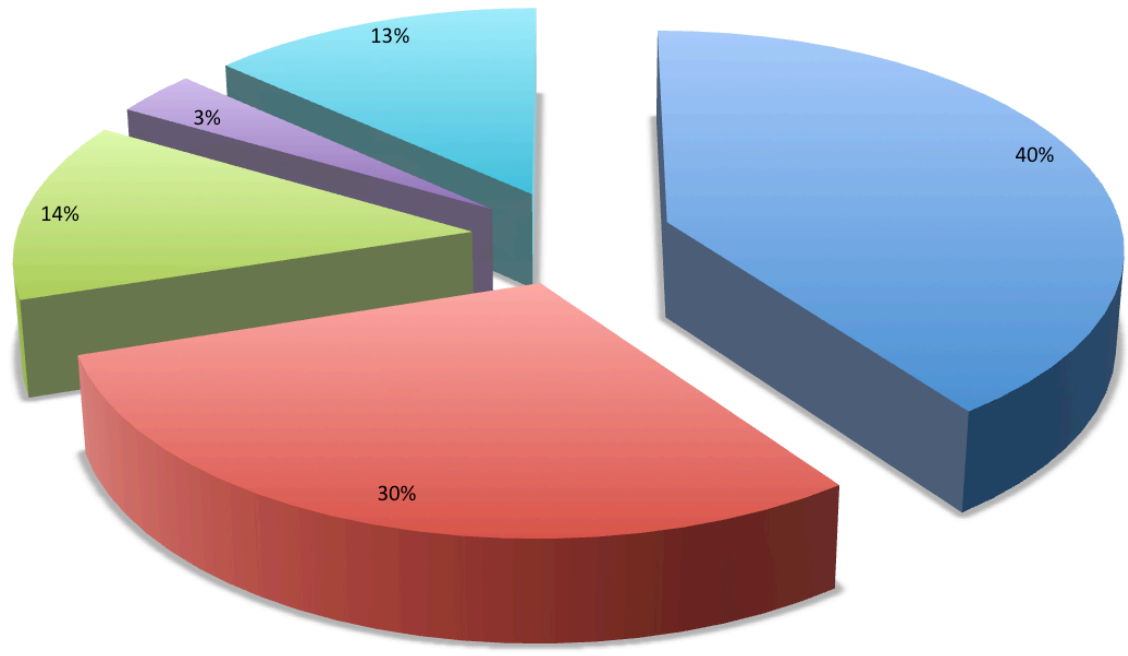

- Nachfolgendes invasives Verfahren

= Nachfolgendes NICHT invasives Verfahren

- Prognostische Neubewertung mit Abbruch der Therapie

= Prognostische Neubewertung Änderung/ oder neue Therapie

Konsil

Abbildung 3: Anteile in Prozent der einzelnen Verfahren an den Konsequenzen, $\mathbf{n}=30$

Als letzter Punkt wurden „Probleme bei der Durchführung“ und „Probleme bei der Beurteilbarkeit" dokumentiert. Wie oben erwähnt, gab es durch einen stark ausgeprägten Meteorismus bei drei Patienten $(4,2 \%)$ Probleme bei der Beurteilbarkeit. Bei der Durchführung von Ultraschalluntersuchungen auf der Palliativstation traten keine Probleme (0) auf. 


\section{Diskussion}

\subsection{Allgemeiner Kontext der Untersuchung}

Ziel der vorliegenden Arbeit war es, die Frage zu beleuchten, welchen Stellenwert diagnostische Maßnahmen im palliativmedizinischen Setting haben. Es sollte erörtert werden, in welchem Ausmaß Diagnostik durchgeführt wurde und welche Konsequenzen für die Patienten resultierten. Die meisten bisher veröffentlichten Studien und Arbeiten beschäftigen sich in diesem Zusammenhang überwiegend mit einer speziellen Fragestellung bzw. diskutieren den Einsatz von Diagnostik bei spezifischen Erkrankungen (z.B. Vitetta et al., 2000; Gishen und Trotman, 2009). Die vorliegende Arbeit sollte unabhängig von der spezifischen Grunderkrankung des Patienten den grundsätzlichen Einsatz von Diagnostik in der Palliativmedizin und die daraus resultierenden Konsequenzen, sowohl auf die Therapie als auch auf das körperliche und psychische Wohl des Patienten bezogen, demonstrieren. Zudem sollten Probleme, die bei oder durch das jeweilige diagnostische Verfahren entstanden sind, dokumentiert werden. Der grundsätzliche Einsatz von Diagnostik, die daraus resultierenden Konsequenzen und die durch die Untersuchungen evtl. entstandenen Probleme sind unabhängig von der spezifischen Erkrankung des Patienten bislang weder systematisch untersucht worden, noch konnten in der Literatur Studien oder Informationen zur Indikation und Formulierung im Hinblick auf Diagnostik bei Palliativpatienten gefunden werden. Mit Hilfe dieser Arbeit sollte gezeigt werden, in welchem Ausmaß Diagnostik in der Palliativmedizin eingesetzt wird und welchen Stellenwert die moderne Diagnostik im palliativmedizinischen Setting hat. Als Grundlage meiner Ausführungen dienten hierzu die durch die Hospiz- und Palliativerhebung (HOPE) gewonnenen Daten aus dem Jahre 2007. Soweit es möglich war, sollte bei diesen Daten und Ergebnissen ebenfalls ein Vergleich zwischen Palliativpatienten auf Palliativstationen und Palliativpatienten auf onkologischen Stationen gezogen werden. Ein weiterer Schwerpunkt dieser Untersuchung lag in der Auswertung und Beurteilung der Daten der stationsintern durchgeführten sonographischen Untersuchungen der Patienten der Palliativstation des Universitätsmedizin Göttingen (UMG), die beispielhaft den Einsatz und die Konsequenzen der Sonographie als bildgebendes und diagnostisches Verfahren in der Palliativmedizin demonstrieren sollten. 


\subsection{Hospiz- und Palliativerhebung (HOPE)}

\subsubsection{HOPE als Patientenregister und Qualitätssicherungsinstrument}

Aus den HOPE- Erhebungsphasen 1999 bis 2007 liegen fast 15000 Patientendatensätze vor (vgl. Anhang 7.3), und im Erhebungszeitraum des Jahres 2007 nahmen mehr als die Hälfte der zu dem Zeitpunkt in Deutschland bestehenden Palliativstationen teil. Die auf Palliativstationen dokumentierten Patienten können daher als repräsentativ für stationär behandelte Palliativpatienten gelten. Die wenigen auf onkologischen Stationen dokumentierten Palliativpatienten der HOPE-Erhebung ähnelten den auf Palliativstationen dokumentierten Palliativpatienten hinsichtlich der fortgeschrittenen Erkrankungen, des Lebensalters und der Liegedauer, so dass ein Vergleich untereinander zulässig ist. Die deutlich geringere Zahl der OS-Patienten im Vergleich zu den PS-Patienten muss bei der Analyse der Ergebnisse zwar als evtl. einschränkend berücksichtigt werden, da es sonst vor allem bei kleinen Datensätzen zu Verzerrungen der Ergebnisse kommen könnte, es verbietet den Vergleich der beiden Einrichtungen aber nicht grundsätzlich. Dass auf onkologischen Stationen weniger Patientenverläufe in HOPE dokumentiert wurden, liegt vermutlich daran, dass sich HOPE primär an palliativmedizinische Versorgungseinrichtungen und Hospize richtet. Auf Stationen mit onkologischem Schwerpunkt stellt die Betreuung von Palliativpatienten nur einen Teilbereich dar, weshalb sich dort die Frage, welcher Patient als Palliativpatient in HOPE dokumentiert werden kann, in stärkerem Maße stellt.

\subsubsection{Teilnehmende Einrichtungen an HOPE und Nutzer des Moduls Diagnostik (DIA)}

Insgesamt wurden im Jahr 2007 durch HOPE 3184 Patientenverläufe dokumentiert, hiervon entfielen 1806 auf klinisch stationär versorgte Patienten (Palliativstationen (PS) und onkologische Stationen (OS), vgl. 4.1.1, Tab.2). Wie im Ergebnissteil schon dargestellt, zeigte die Auswertung der Daten, dass der überwiegende Teil der zurückgesandten DIA-Module $(86,1 \%)$ von den beiden stationären Einrichtungen stammte (vgl. Kapitel 4.1). Daher beziehe ich mich im Sinne der besseren Vergleichbarkeit im weiteren Diskussionsteil ausschließlich auf die genannten klinischstationären Einrichtungen. Zudem erhielten die in Hospizen betreuten Patienten keine 
Diagnostik und müssen somit hier im Weiteren nicht berücksichtigt werden. Ein weiterer Grund, warum ich mich ausschließlich auf die stationären Einrichtungen beziehe, ist die Verfügbarkeit der diagnostischen Mittel. Im stationären Bereich ist davon auszugehen, dass alle in HOPE abgefragten diagnostischen Verfahren ohne lange Wegezeiten verfügbar und durchführbar sind. Im Gegensatz zu den an ein Krankenhaus angeschlossenen PS und OS ist es für die dokumentierten ambulanten Einrichtungen mit einem viel höheren zeitlichen, logistischen und ggfls. finanziellen Aufwand verbunden, die abgefragten diagnostischen Untersuchungen durchzuführen. Um die daraus evtl. entstehende Verfälschung der Ergebnisse auszuschließen, wurden die ambulanten Einrichtungen für meine Fragestellung im Hinblick auf die Nutzung, die Relevanz und die durch Diagnostik entstandenen Probleme ausgeschlossen.

\subsubsection{Demographische Daten}

Die demographischen Daten zeigten, dass der Einsatz von Diagnostik in den verschiedenen stationären palliativmedizinischen Einrichtungen nicht altersabhängig ist. Das Durchschnittsalter des HOPE-Patientenkollektivs betrug 66,3 Jahre, das durchschnittliche Alter der HOPE-Patienten mit DIA-Modul 67,9 Jahre. Es zeigte sich hier, dass Diagnostik nicht, wie man vielleicht vermuten würde, eher den jüngeren Patienten zukommt. Diagnostik wurde demnach bei Palliativpatienten unabhängig vom Alter eingesetzt. $\mathrm{Zu}$ diesem Ergebnis kommt man auch, wenn man einen Vergleich zwischen Palliativpatienten auf den onkologischen und den Patienten auf Palliativstationen zieht. Das Alter zwischen onkologisch betreuten Patienten und Patienten von Palliativstationen mit DIA zeigte keine signifikante Abweichung (PS: 67,9 Jahre vs. OS: 67,6 Jahre). Die Geschlechterverteilung fiel sowohl bei den Patienten mit DIA-Modul (PS: weibl. 55,5\%/ männl. 44,5\% vs. OS: weibl. 51,9\%/ männl. 48,1\%) als auch ohne DIA-Modul (PS: weibl. 53,5\%/ männl. 46,5\% vs. OS: weibl. 50,7\%/ männl. 49,3\%) leicht zugunsten weiblicher Teilnehmer aus. Der leichte Überhang weiblicher Patienten lässt sich auch im HOPE-Gesamtkollektiv nachvollziehen und ist am ehesten auf demographische und epidemiologische Kriterien zurückzuführen. Es steht jedoch auch zu vermuten, dass es bei Erkrankung einer Ehefrau rascher zu psychosozialem Unterstützungsbedarf kommt als bei der Erkrankung eines Ehemannes, der in konventionellen Rollenmodellen zumeist noch längere Zeit durch die Ehefrau versorgt 
wird, ehe palliativmedizinische Hilfe aufgesucht wird. Letztendlich verbleiben die Gründe für den Überhang weiblicher Patientinnen in der HOPE-Dokumentation spekulativ.

Die Auswertung zeigte auch, dass es durch den Einsatz von Diagnostik zu keiner relevanten Liegezeitverlängerung kam. Auf Palliativstationen betrug die durchschnittliche Verweildauer 13,1 Tage, egal ob die Patienten eine diagnostische Maßnahme erhielten oder nicht. Auf onkologischen Stationen waren es 13,6 Tage bei den Patienten ohne Diagnostik und 13,2 Tage bei den Patienten mit ausgefülltem DIAModul. Der Vergleich der Liegedauer zwischen den beiden Einrichtungen ergab keinen signifikanten Unterschied. Dieses Ergebnis ist insofern von Bedeutung, als dass diagnostische Maßnahmen demnach nicht dem palliativmedizinisch relevanten Ziel einer möglichst weitreichenden häuslichen Versorgung entgegen stehen.

\subsubsection{Diagnostik-Modul (DIA)}

Das Modul Diagnostik diente zur speziellen Erfassung zum Einsatz von nichtinvasiver Diagnostik in der Palliativmedizin. Aus den in Kapitel 5.2.2 erwähnten Gründen bezieht sich die folgende Diskussion nur auf die beiden stationären Bereiche. Auch wenn bei dem Einsatz und der Befragung durch dieses Modul keine wissenschaftlich korrekte Randomisierung der Patienten stattgefunden hat, besteht aus den in 5.1.1-5.2.3 genannten Gründen eine weitgehende Strukturgleichheit und eine weitgehende Homogenität des Patientenkollektives der HOPE-Erfassung. Die Tatsache, dass die Teilnahme am DIA-Modul gezielt angemeldet und eingefordert werden musste, lässt schließen, dass in diesen Einrichtungen die Motivation zur Teilnahme an einer wissenschaftlichen Studie groß war und die ausfüllenden Personen mit Sorgfalt dokumentiert haben; allerdings kann daher nicht von einer uneingeschränkten Repräsentativität der Stichprobe ausgegangen werden.

Es bleibt die Frage offen, warum für die restlichen an HOPE teilnehmenden Patienten kein DIA-Modul dokumentiert wurde. Einerseits kann die Tatsache, dass die Bögen nicht ausgefüllt wurden, das Fehlen von Diagnostik bedeuten. Dies ist aber sehr unwahrscheinlich, da von den 595 dokumentierten Patienten 73\% (437 Patienten) mindestens ein diagnostisches Verfahren hatten (vgl. 4.1.1, Tab.2 und 4.1.3, Tab.4). 
Somit besteht die Vermutung, dass nicht jede durchgeführte Diagnostik dokumentiert wurde und es bleibt zu diskutieren, warum dies der Fall ist. Ein Grund könnte ein Zeitmangel im ärztlich-klinischen Umfeld sein. Der Zeitfaktor wurde auch schon in einer anderen Studie von André et al. als wichtiger Faktor für die Nichtteilnahme an Studien und/ oder Forschung beschrieben (André et al., 2009). Ein weitere Einflussgröße für die fehlende Teilnahme an dem DIA-Modul könnten eventuelle Verständnisprobleme, die Einstellung der Mitarbeiter oder mangelnde Motivation sein, welche ebenfalls schon in einigen Studien beschrieben wurden (Jordhoy et al., 1999; Ling et al., 2000; André et al., 2009).

\subsubsection{DIA-Modul Teil I: Mikrobiologie}

Ingesamt hatten von den 437 Patienten, bei denen mindestens ein diagnostisches Verfahren dokumentiert wurde (vgl. 4.1.3, Tab.4), 155 eine mikrobiologische Analyse. Die schon im Ergebnissteil beschriebene Diskrepanz im Hinblick auf die Einsatzhäufigkeit zwischen den beiden Einrichtungen (vgl. 4.1.4) könnte mit dem auf onkologischen Stationen häufiger als auf Palliativstationen eingesetzten Chemotherapien (Radbruch et al., 2002) in Zusammenhang stehen. Die Nebenwirkungen der antineoplastischen Therapie (z.B. Granulozytopenie (Neutropenie), Granulozytenfunktionsstörung, T-Zellsuppression, etc.) führen häufig zu einer erhöhten Infektanfälligkeit (Maschmeyer, 2005), was wiederum den häufigeren Einsatz von mikrobiologischen Verfahren erklären würde. Im Gegensatz dazu stehen bei den Patienten der Palliativstation eher Mangelernährung, Asthenie, Vigilanzminderung, Immobilität und Barrierefunktionsstörungen als Risikofaktoren für Infektionen im Vordergrund (Pereira, 1998; Homsi et al., 2000; Vitetta et al., 2000; Ripamonti und Fusco, 2002). Diese sind mit einem weniger hohen Risiko für schwerere Infektionen behaftet, was den geringeren Einsatz von mikrobiologischen Verfahren erklären würde. Im Allgemeinen sind Infektionen häufige Begleiterkrankungen bei Palliativpatienten (Pestinger et al., 2004). Je nach Studie wurde bei 40-55\% der Patienten mit weit fortgeschrittenen Tumorleiden eine Infektion nachgewiesen (Pereira et al., 1998; NagyAgren und Haley, 2002). Grundsätzlich wird der Einsatz von Antibiotika kontrovers diskutiert. In einigen Studien wird eine antibiotische Therapie als wenig sinnvoll oder sogar als zu aggressive Maßnahme beschrieben (Sorbye, 2000). In anderen Studien wird 
der Einsatz von Antibiotika als Teil der Symptomkontrolle gesehen (Vitetta et al., 2000). Die in der Literatur stark divergierenden Meinungen zum Einsatz von Antibiotika in der Palliativmedizin und das Fehlen von einheitlichen Outcomekriterien zur Erfolgskontrolle (Pestinger et al., 2004) zeigen, dass es eine große Unsicherheit im Einsatz von Antibiotika bei Palliativpatienten gibt. Dies könnte eine weitere Erklärung für die unterschiedlichen Ergebnisse der in HOPE dokumentierten Einrichtungen sein. Insgesamt wurde für 88,4\% (vgl. 4.1.5, Tab.5) aller durchgeführten mikrobiologischen Untersuchungen eine therapeutische Konsequenz dokumentiert, mit starker Abweichung im Vergleich zwischen beiden Einrichtungsarten. Auf Palliativstationen hatten 90,5\% der Untersuchungen eine therapeutische Konsequenz, auf onkologischen Stationen nur 42,9\%. Wenn es zu einer therapeutischen Konsequenz kam, handelte es sich allerdings bei beiden Einrichtungen um die „Verordnung einer Antibiose“ (vgl. Tab.5). Aus diesem Ergebnis lässt sich schlussfolgern, dass das Ziel der mikrobiologischen Untersuchung bei beiden am ehesten die Einleitung einer kalkulierten antibiotischen Therapie war. Die Autoren in der Studie von Vitetta et al. (2000) kamen zu dem Schluss, dass ein adäquates Infektionsmanagement zu einer besseren palliativen Symptomkontrolle führt. Andere Studien über den Einsatz von Antibiotika in der Palliativmedizin kommen ebenfalls zu dem Schluss, dass eine gezielte Therapie im Vergleich zur empirischen Antibiotikatherapie mit einer Lebenszeitverlängerung assoziiert ist (Lam et al., 2005; Reinbolt et al., 2005). In Zusammenhang mit diesen Studien gesehen, die belegen, dass der kalkulierte Einsatz einer antimikrobiellen Therapie bei Palliativpatienten von Vorteil ist und zur Linderung belastender Symptome und zu einer Lebensverlängerung führen kann (Vitetta et al., 2000; Lam et al., 2005; Reinbolt et al. ,2005), haben mikobiologische Verfahren in der Palliativmedizin durchaus ihre Berechtigung und sollten bei gegebener Indikationsstellung zur Diagnostik eingesetzt werden.

\subsubsection{DIA-Modul Teil II: Bildgebung}

Die Analyse der Daten zeigte, dass 69,1\% der insgesamt 595 Patienten der beiden Einrichtungen mindestens ein bildgebendes Verfahren $\mathrm{zu}$ diagnostischen Zwecken erhielten. In Durchschnitt wurden 1,7 Verfahren pro Patient angewendet. In beiden Einrichtungen waren die Sonographie und die konventionelle Röntgendiagnostik die am 
häufigsten angewandten Verfahren, darauf folgten die Schichtbildverfahren (CT/ MRT) und an letzter Stelle die nuklearmedizinischen Verfahren (NUK) (vgl. 4.1.6, Tabelle 6). In der Einsatzhäufigkeit von Bildgebung zum Zweck der Diagnostik gab es zwischen den beiden Einrichtungen keinen signifikanten Unterschied (vgl. Kapitel 4.1.6). Lediglich die Gewichtung unter den einzelnen Verfahren unterschied sich leicht voneinander. So wurde auf Palliativstationen die Sonographie am häufigsten angewandt, gefolgt von der konventionellen Röntgenuntersuchung. Auf onkologischen Stationen war dieses Verhältnis umgekehrt. Auffallend war, dass onkologische Stationen signifikant mehr nuklearmedizinische und Schichtbildverfahren durchführten, was vermutlich im Zusammenhang mit Staginguntersuchungen und Fragen zur Tumortherapie zu sehen ist. Dies steht auch in Einklang mit den Ergebnissen einer noch nicht veröffentlichen Untersuchung aus der Abteilung Palliativmedizin der Universitätsmedizin Göttingen (Pache, 2010), welche darauf hinweisen, dass auf onkologischen Stationen (häufiger als auf Palliativstationen) Tumortherapien zum Zwecke der Tumorgrößenreduktion eingesetzt werden, was die Notwendigkeit von Schichtbilduntersuchungen zur Tumorgrößen- bzw. Volumenkontrolle nach sich zieht.

Die Sonographie eignet sich eher bei Fragestellungen wie Dyspnoe, Obstruktion usw., hier gibt sie oft schon ausreichend Aufschluss über die Ursache und damit über Therapieoptionen. Dass die Bildgebung Therapieentscheidungen unterstützt, zeigte die Datenauswertung des Unterpunktes „Therapeutische Konsequenzen“. 80,6\% der bildgebenden Untersuchungen folgte mindestens eine therapeutische Konsequenz. Zwei der drei häufigsten Konsequenzen war ein nachfolgendes invasives (Punktion, Endoskopie, Blasenkatheter, etc.) oder nichtinvasives Verfahren (medikamentöse Ileustherapie, Diuretika, etc.), was die Bedeutung der Bildgebung für die Symptomkontrolle belegt. Die letzte der drei häufigsten Konsequenzen war eine „generelle prognostische Neubewertung“ (vgl. 4.1.6, Tab.7). Auch hier zeigt sich der Stellenwert der Bildgebung für die Statuserhebung und den dynamischen Prozess, in dem sich der Palliativpatient auf Grund seiner Erkrankung befindet. Eine Herausforderung in der Palliativmedizin ist die ständige Änderung und Neudefinition der individuellen Therapieziele des Patienten (Samonigg et al., 2000; Pestinger et al., 2004), wobei die Bildgebung eine wichtige Hilfe sein kann. Leider lassen die Daten keine Aussage darüber zu, welches der durchgeführten bildgebenden Verfahren zu welchen therapeutischen Konsequenzen geführt hat oder ob, falls es mehrere gab, welche in 
welcher Reihenfolge stattgefunden haben. Es bleibt $\mathrm{zu}$ vermuten, dass hier erfahrungsbezogen vorgegangen wurde, d.h. dass z.B. bei dem Verdacht auf einen Ileus zuerst eine Sonographie des Abdomen und dann eine röntgenologische AbdomenÜbersicht erfolgte.

\subsubsection{DIA-Modul Teil II: Probleme bei der Durchführung und Beurteilung}

Ein wichtiges Ziel der Studie war die Dokumentation der Probleme im Zusammenhang mit der Durchführung von Diagnostik bei Palliativpatienten. Neben den Problemen, die durch die Durchführung der Untersuchung entstanden sind, konnte die dokumentierende Person auch Probleme angeben, die eventuell die Beurteilung erschweren, wie z.B. Meteorismus. Hier zeigte sich eine deutliche Diskrepanz in der Häufigkeit der Probleme zwischen den beiden Einrichtungen. Auf onkologischen Stationen wurden signifikant mehr Problemen als auf Palliativstationen dokumentiert. Hier waren bei mehr als der Hälfte der Patienten (56\%) Probleme angegeben worden. Auf beiden Stationen waren die häufigsten Probleme bei der Durchführung: Schmerzen durch die Untersuchung, Transport- und Lagerungsprobleme und Ruhebedürfnis des Patienten (vgl. Kapitel 4.1.7). Die durch das Ergebnis aufgeworfene Frage nach der Ursache dieser Diskrepanz lässt sich durch die vorliegenden Daten nicht beantworten. Die dokumentierten Probleme jedoch sind häufige Probleme von Palliativpatienten, unabhängig davon durch welche Einrichtung sie betreut werden oder welches diagnostisch/ therapeutische Verfahren sie bekommen.

\subsubsection{DIA-Modul I+II: „technisch-analytisches Ergebnis“}

Die Abschnitte „Mikrobiologische Verfahren“ (DIA-Modul Teil I) und „Bildgebende Verfahren“ DIA-Modul Teil II) konnten am Ende jeweils hinsichtlich ihres „technischanalytischen Ergebnisses“ bewertet werden. Die dokumentierende Person konnte hier Angaben machen, ob bei der jeweiligen Untersuchung ein verwertbares Ergebnis zu erzielen war (Einfachauswahl „Ja / Nein“). Im Bezug auf die praktische Durchführung fiel hier eine Unterdokumentation auf, die eine verlässliche Interpretation der Ergebnisse nur eingeschränkt zuließ. So gibt es bis auf eine Auswertung in allen anderen das Phänomen, dass es im Vergleich weniger technisch-analytisch verwertbare 
Ergebnisse gibt als therapeutische Konsequenzen (vgl.4.1.6, Tab.7). Im Durchschnitt lassen ein Viertel der Bögen offen, ob es ein verwertbares Ergebnis gegeben hat oder nicht.

Dieses Dokumentationsergebnis widerspricht nicht der Annahme, dass die bildgebenden Verfahren ein gutes Instrument zum Zweck der Diagnostik sind und häufig ein verwertbares Ergebnis haben. Selbst wenn alle nicht bewerteten Verfahren mit „Nein“ bewertet worden wären, wären die Ergebnisse der abgefragten diagnostischen Verfahren hinsichtlich ihres „technisch-analytischen Ergebnisses“ immer noch ausreichend überzeugend und ein Beweis für die Qualität des Einsatzes von Diagnostik.

Da hier vermutlich ein methodisches Problem durch den Fragebogen vorliegt, wäre zu überlegen, ob man diese Schwäche des Erhebungsbogens bei zukünftigen Untersuchungen durch z.B. einen Pretest-Bogen, in dem gezielt diese Problematik Beachtung findet, verringern kann.

\subsection{Sonographien der Palliativstation der Universitätsmedizin Göttingen}

\subsubsection{Allgemeines und Hintergrund der Studie}

Die im Rahmen der prospektiven Studie auf der Palliativstation des Universitätsklinikums in Göttingen (UMG) durchgeführten 70 Ultraschalluntersuchungen wurden wie im Methodikteil beschrieben (vgl. 3.2) durchgeführt und dokumentiert. Ziel dieses Teils der Studie war es, den Stellenwert der Sonographie in der palliativmedizinischen Diagnostik, die therapeutischen Konsequenzen und die Probleme, die durch die Untersuchung für den Patienten entstanden sind, zu erheben. Nach der Auswertung erfolgte ein Vergleich mit den aus dem Jahre 2007 gewonnenen Daten der Auswertung des DIA-Moduls Abschnitt II (Bildgebung). Das Ziel dieser Gegenüberstellung war es, Aussagen über die Relevanz von Sonographie als alleiniges bildgebendes Verfahren machen zu können. Zum Zweck der besseren Vergleichbarkeit wurde eine Exceltabelle entwickelt, die sich sehr stark an das DIA-Modul des HOPE Fragebogens anlehnt (vgl. Anhang 7.4). Weitere Gründe für die Entscheidung, die Sonographie als bildgebendes Verfahren zu wählen, waren die 
geringen Kosten der Untersuchung, der geringe Aufwand, die schnelle Durchführbarkeit, die vermutlich geringe Belastung für den Patienten, die flächendeckende Verfügbarkeit und die Repräsentativität auch im hausärztlichen Bereich (vgl. Anhang 7.3.2). Diese Punkte werden im Weiteren wieder aufgegriffen und im Zusammenhang mit den Ergebnissen diskutiert. Ein wichtiger Unterschied zu den im DIA-Modul dokumentierten Untersuchungen war die Tatsache, dass die Sonographien auf der Station mit einem mobilen stationseigenen Sonographiegerät, das jeder Zeit verfügbar war, durchgeführt wurden.

\subsubsection{Demographische Daten und Verweildauer der UMG-Patienten}

Die demographischen Daten differierten nur gering von denen des DIA-Kollektives. Die Patienten der UMG hatten eine durchschnittliche Liegedauer von 13,4 Tagen und ein durchschnittliches Alter von 61,3 Jahren. Die Geschlechterverteilung war auch hier mit $54,3 \%$ weiblichen und 45,7\% männlichen Patienten relativ ausgewogen. Eine Selektion oder Einteilung in die verschiedenen palliativmedizinisch gebräuchlichen Stadien nach Jonen-Thielemann (Johnen-Thielemann, 2007) und eine darauffolgende Auswahl der Patienten oder eine Selektion anhand spezieller Fragestellungen erfolgte nicht, um ähnliche Bedingungen wie bei dem ausgewerteten DIA-Modul zu erzeugen. Die Auswertung der eigenen Daten zeigte, dass auch hier, wie bei der Auswertung des DIAModuls, dass Sonographie unabhängig vom Alter und Geschlecht durchgeführt werden kann. Ebenfalls zeigten die Daten der Patienten aus Göttingen, im Vergleich mit den Daten des HOPE Kollektivs, dass die Sonographie zum Zweck der Diagnostik zu keiner relevanten Liegezeitverlängerung führt (Liegezeit: HOPE PS 13,1 Tage).

\subsubsection{Auswertung der UMG-Patienten}

Bei drei der 70 Patienten konnte die Untersuchung auf Grund von stark ausgeprägtem Meteorismus nicht ausgewertet werden. Bei den restlichen 67 Patienten gab es weder Probleme in der Beurteilbarkeit noch bei der Durchführung. Dieses Ergebnis lässt den Schluss zu, dass sich die im DIA-Modul dokumentierten Probleme durch Flexibilität im Einsatz und kleine bzw. keine Wege stark minimieren lassen. Die Durchführung der Ultraschalluntersuchung auf der Palliativstation ermöglichte es, die Untersuchungen 
zeitlich und örtlich den Bedürfnissen und dem Zustand des Patienten anzupassen. Das hatte wiederum eine geringere physische und psychische Belastung des Patienten zu Folge und führte zu weniger Problemen. Zu diesem Ergebnis kamen auch Gishen und Trotman in ihrer Studie „Bedside ultrasound - experience in a palliative care unit“ (2009) und Gehmacher (2006). Gishen und Trotman stellten in ihrer Studie sogar die These auf, dass es durch den stationsinternen Einsatz der Sonographie zu einer Liegezeitverkürzung kommen könnte. Ihr Argument zu dieser These war folgendes: Wegfall von evtl. lange dauernden Wegen zwischen den einzelnen Abteilungen und daraus resultierende geringe Wartezeiten. $\mathrm{Zu}$ demselben Ergebnis kamen auch Gehmacher et al. (2006) der in seiner Studie die Daten von 478 Patienten dokumentierte. Was die Autoren in ihrer Studie ebenfalls feststellten, war, dass der flexible zeitliche Einsatz des Geräts mit Vorteilen für die Patienten verbunden ist. So sollten die geplanten Sonographien bei der Aufnahme oder zu einer Gelegenheit, die für den Patienten angenehm ist, stattfinden. Somit war es möglich, die Untersuchungstermine den Bedürfnissen und dem Zustand des Patienten anzupassen. Ein weiterer wichtiger Faktor laut Gehmacher ist die Tatsache, dass die Untersuchung von dem Stationsarzt den die Patienten kennen und vertrauen durchgeführt wird und somit zu einer höheren Sicherheit und einem größeren psychischen Wohlbefinden der Patienten führt (Gehmacher, 2006).

In dem Punkt „therapeutische Konsequenzen“ wurde der stationsinterne Bogen im Vergleich zu dem DIA-Modul in den Antwortoptionen etwas modifiziert und erweitert. Es wurde, anders als in dem DIA-Modul, nicht nur erhoben, ob es zu einer prognostischen Neubewertung gekommen war, sondern auch ob es in diesem Zusammenhang zu einer Änderung des Therapieziels oder evtl. sogar zu einem Abbruch der aktuellen Therapie kam. Ebenfalls wurde der Punkt „Konsil“ eingeführt, durch den dokumentiert werden konnte, ob im Anschluss an die Sonographie eine zweite Fachrichtung hinzugezogen wurde.

Der Stellenwert der Sonographie widerspiegelt sich nicht nur in ihrer diagnostischen Wertigkeit, sondern auch in ihrer Bedeutung für die Symptomkontrolle aufgrund der sich anschließenden therapeutischen Maßnahme, die bei einem Drittel der Patienten therapeutische Konsequenzen nach sich zogen. Als häufigste Konsequenz wurde ein anschließendes therapeutisches Verfahren wie z.B. eine Aszitespunktion, 
Abführmaßnahmen usw. angegeben. Durch die schnelle Verfügbarkeit der Sonographie und der daraus folgenden unmittelbaren Befundung können weitere schwere Komplikationen möglicherweise verhindert und Beschwerden gelindert werden (Mariani und Setla, 2010). Insgesamt hatten 7,1\% der Untersuchungen Einfluss auf die prognostische Einschätzung der Patienten. Bei der Auswertung des DIA-Moduls (Bildgebende Verfahren) zeigte sich eine deutlich höhere Relevanz der bildgebenden Verfahren im Hinblick auf die prognostische Einschätzung. Mehr als ein Viertel der durchgeführten und mit Hilfe des DIA-Moduls dokumentierten bildgebenden Verfahren wurden als prognostisch relevant bewertet. Mittels der Sonographie lassen sich z.B. neue Metastasen, Lymphknotenschwellungen, oder Größenveränderungen des Tumors gut beurteilen. Die Sonographie stößt jedoch bei konkreteren Fragestellungen wie z.B. Staging oder Vitalitätsnachweise an ihre Grenzen, so dass weitere schichtbildgebende oder nuklearmedizinische Verfahren auch im Palliativsetting erforderlich sein können. Hier bildet die Sonographie als wenig belastendes und einfaches Verfahren die Basis für die Entscheidung bezüglich einer weiteren aufwändigeren Diagnostik. Vier Patienten wurden konsilarisch $\mathrm{zu}$ anderen Fachrichtungen überwiesen (Urologie: 3 Patienten, Radiologie: 1 Patient). Im Vergleich zu den Ergebnissen des DIA-Moduls, in dem nur die Summe der bildgebenden Verfahren hinsichtlich ihrer therapeutischen Relevanz bewertet werden konnte, ist das Ergebnis der Sonographie als alleinige bildgebende Untersuchung relativ hoch (DIA: 80,8; UMG: 37,2\%).

\subsection{Schlussfolgerungen}

Sowohl aus den Daten des DIA-Moduls der HOPE-Erhebung als auch aus den prospektiv gewonnenen Daten der Palliativstation Göttingen können folgende Schlussfolgerungen gezogen werden:

- Diagnostik wird unabhängig von Alter oder Geschlecht des Patienten eingesetzt und führt zu keiner signifikanten Liegezeitverlängerung bei Palliativpatienten.

- Sowohl mikrobiologische als auch bildgebende Verfahren werden zu diagnostischen Zwecken mit hoher therapeutischer Relevanz in der Palliativmedizin eingesetzt. Der Einsatz von mikrobiologischen Verfahren ermöglicht eine kalkulierte antibiotische Therapie häufig mit einem 
therapeutischen Ziel oder dem Ziel einer besseren Symptomkontrolle. Mehr als zwei Drittel der Patienten mit dokumentierter Diagnostik erhielten mindestens ein bildgebendes Verfahren. Die häufigsten daraus resultierenden therapeutischen Konsequenzen waren ein nachfolgendes invasives oder nicht invasives Verfahren und die prognostische Neubewertung. Dies verdeutlicht die Bedeutung der Bildgebung in Bezug auf den dynamischen Krankheitsprozess, in dem sich der Palliativpatient befindet, und die Symptomkontrolle. Der Einsatz von Diagnostik führt bei den wenigsten Patienten $\mathrm{zu}$ weiteren physischen Belastungen und ist mit einem vertretbaren Ausmaß an Problemen verbunden.

- Die Sonographie ist vielseitig zu diagnostischen wie aber auch zu therapeutischen Zwecken, z.B. bei Punktionen einsetzbar. Bei mehr als einem Drittel der Patienten führte die Sonographie als alleiniges diagnostisches Verfahren zu therapeutisch wichtigen Konsequenzen, wie prognostischer Neubewertung oder weiteren invasiven / nichtinvasiven Verfahren. Probleme, die durch die Belastungen der Untersuchung möglicherweise entstehen könnten, lassen sich durch differenzierte Indikationsstellung, Flexibilität in der Durchführung und im Einsatz so gut wie ganz vermeiden. Die Ergebnisse zeigen, dass die Sonographie als diagnostisches Mittel ihren Stellenwert auch im palliativmedizinischen Setting hat. Hier könnte die Sonographie einen Kompromiss zwischen zu exzessiver und gar keiner apparativen Untersuchung sein.

- Durch die Ergebnisse der durchgeführten Untersuchungen ergeben sich neue Aspekte zur palliativmedizinischen Praxis und zur bisherigen low-technologyHaltung in der Palliativmedizin: ein „Palliativpatient“ charakterisiert sich nicht durch das Fehlen von Diagnostik oder Tumortherapie, sondern durch die besondere Intention und Indikation und die veränderte Gewichtung, die man seinen diagnostischen und therapeutischen, durch viele Berufsgruppen getragenen Maßnahmen einräumt, um das Ziel der Palliativmedizin, Linderung von körperlichen, psychischen, sozialen und spirituellen Symptomen, zu erreichen. 


\section{Zusammenfassung}

Die große Anzahl der zur Verfügung stehenden modernen diagnostischen nichtinvasiven Verfahren und der medizinische Fortschritt allgemein werfen folgende Fragen auf: Wie viel Diagnostik braucht ein Palliativpatient? Wie belastend ist Diagnostik für einen Palliativpatienten und welcher Benefit entsteht durch den Einsatz von Diagnostik? Zu der Problematik, inwieweit eine konservative low-technology-Haltung in der modernen Palliativmedizin noch berechtigt ist, existieren bisher jedoch nur wenige Daten, ebenso über die Einsatzhäufigkeit, die therapeutischen Konsequenzen und die Belastung die durch den Einsatz von Diagnostik entstehen. Im Rahmen der Hospiz- und Palliativerhebung (HOPE) 2007 wurden daher mit Hilfe des Moduls Diagnostik (DIA) in ambulanten und stationären bzw. in onkologischen, palliativmedizinischen Einrichtungen und Hospizen Aspekte zur Quantität und Qualität von bildgebenden und mikrobiologischen Verfahren erhoben. Es wurde der Einsatz und die therapeutische Relevanz von nichtinvasiver Diagnostik und die durch ihren Einsatz entstandenen Probleme erfasst. Da 86,1\% aller DIA-Module im stationären Bereich (Palliativstationen (PS), onkologische Stationen (OS)) ausgefüllt wurden, wurden im Rahmen der besseren Vergleichbarkeit nur die Ergebnisse dieser 595 Patienten (PS: 565, OS: 30) betrachtet und ausgewertet. Hinsichtlich ihrer demographischen Daten und der Liegedauer gab es zwischen den beiden stationären Einrichtungen keinen signifikanten Unterschied, ebenso war kein relevanter Unterschied zwischen dem Patientenkollektiv mit und ohne DIA Modul festzustellen (Alter: HOPE 67,8 Jahre, DIA 67,9 Jahre; Verweildauer: HOPE 18,2d, DIA 16,9d). Von den dokumentierten diagnostischen Maßnahmen entfielen $26,1 \%$ aller Verfahren auf mikrobiologische Laboruntersuchungen, die in $86,5 \%$ als therapeutisch relevant bewertet wurden (z.B. im Bezug auf eine antibiotische Therapie). Bei den bildgebenden Verfahren hatten 411 Patienten mindestens ein bildgebendes Verfahren, wobei die Sonographie und die konventionelle Röntgendiagnostik die am häufigsten angewandten Verfahren waren, gefolgt von den schichtbildgebenden- und nuklearmedizinischen Verfahren. 80,8\% aller bildgebenden Untersuchungen wurden als therapeutisch relevant eingeschätzt. Die häufigsten Konsequenzen waren ein sich anschließendes therapeutisches Verfahren. Schmerzen, Transportprobleme und Müdigkeit nach der Untersuchung waren die am häufigsten dokumentierten untersuchungsassoziierten Belastungen. 
Anhand von 70 in der eigenen Abteilung Palliativmedizin der UMG durchgeführten Sonographien konnte beispielhaft der Einsatz der Sonographie als alleiniges nichtinvasives Verfahren dokumentiert werden. Bei diesen Patienten betrug das durchschnittliche Alter 61,3 Jahren und die mittlere Verweildauer lag bei 13,4d. Bei $37,2 \%$ der Patienten hatte die sonographische Untersuchung mindestens eine therapeutisch relevante Konsequenz. Wie auch in der HOPE-Dokumentation war hier ein weiteres invasives und/oder nichtinvasives therapeutisches Verfahren die häufigste Konsequenz. Probleme durch die Untersuchung selbst wurden nicht berichtet.

Die Auswertung zeigt, dass Diagnostik unabhängig vom Alter durchgeführt wird und zu keiner Liegezeitverlängerung führt. In der stationären Palliativmedizin kann Diagnostik sowohl bei prognostischen wie auch bei therapeutischen Fragestellungen relevant und hilfreich eingesetzt werden. Der Einsatz der Sonographie als alleiniges nichtinvasives Verfahren führt oft schon zu therapeutisch relevanten Konsequenzen. Sie kann zu einer Klärung der Symptomherkunft dienen oder zu weiteren gezielten therapeutischen Interventionen führen und damit indirekt eine symptomorientierte Therapie unterstützen und verbessern.

Zusammenfassend lässt sich feststellen, dass diagnostische Verfahren auch im besonderen klinischen Bereich der Palliativmedizin ihren berechtigten Stellenwert haben. Im Idealfall hilft das Ergebnis der diagnostischen Untersuchung bei der Symptomlinderung oder der Therapiefindung, ohne den Patienten zu sehr zu belasten. Es konnte erstmals für die Palliativmedizin gezeigt werden, dass bereits der alleinige Einsatz der Sonographie zu therapeutisch relevanten Konsequenzen führt. Der Einsatz von sog. „bedside ultrasound“ hilft, die Belastung für den Patienten so gering wie möglich zu gestalten, ist schnell durchzuführen und jederzeit wiederholbar. Auch in finanzieller Hinsicht bietet die bedside ultrasound viele Vorteile und kann helfen, Kosten einzusparen. Vor jedem diagnostischen Verfahren sollte jedoch immer hinterfragt werden, ob das Verfahren indiziert ist und welche Konsequenzen für den Patienten daraus folgen, denn nur ein gezielter und überlegter Einsatz von Diagnostik kann zu einer Symptomlinderung und/oder einer Anpassung der Behandlung im palliativmedizinischen Setting führen. 


\section{Anhang}

\subsection{Dokumentationsbögen}

\subsubsection{Hospiz- und Palliativerhebung (HOPE) Basisbogen}

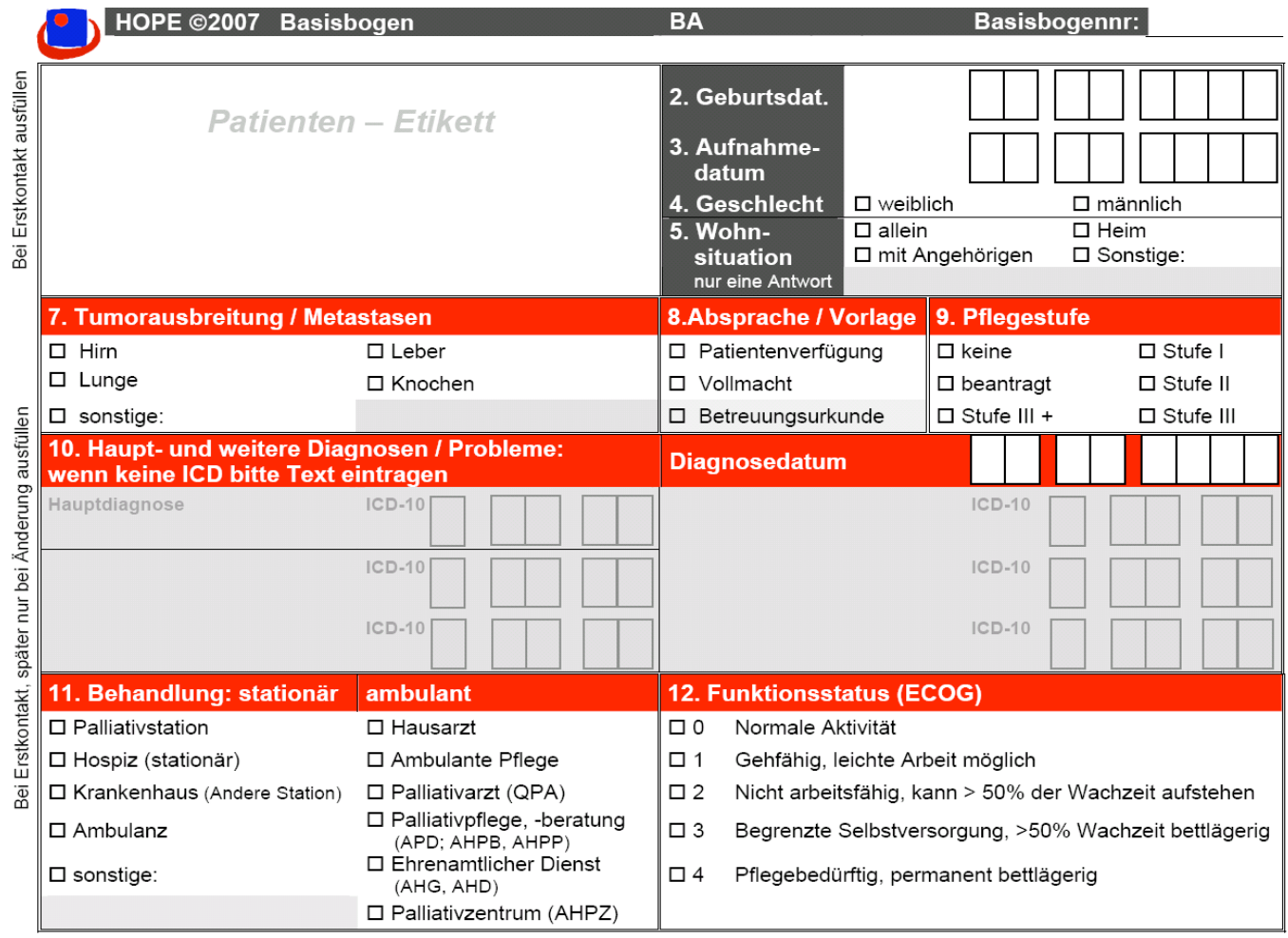

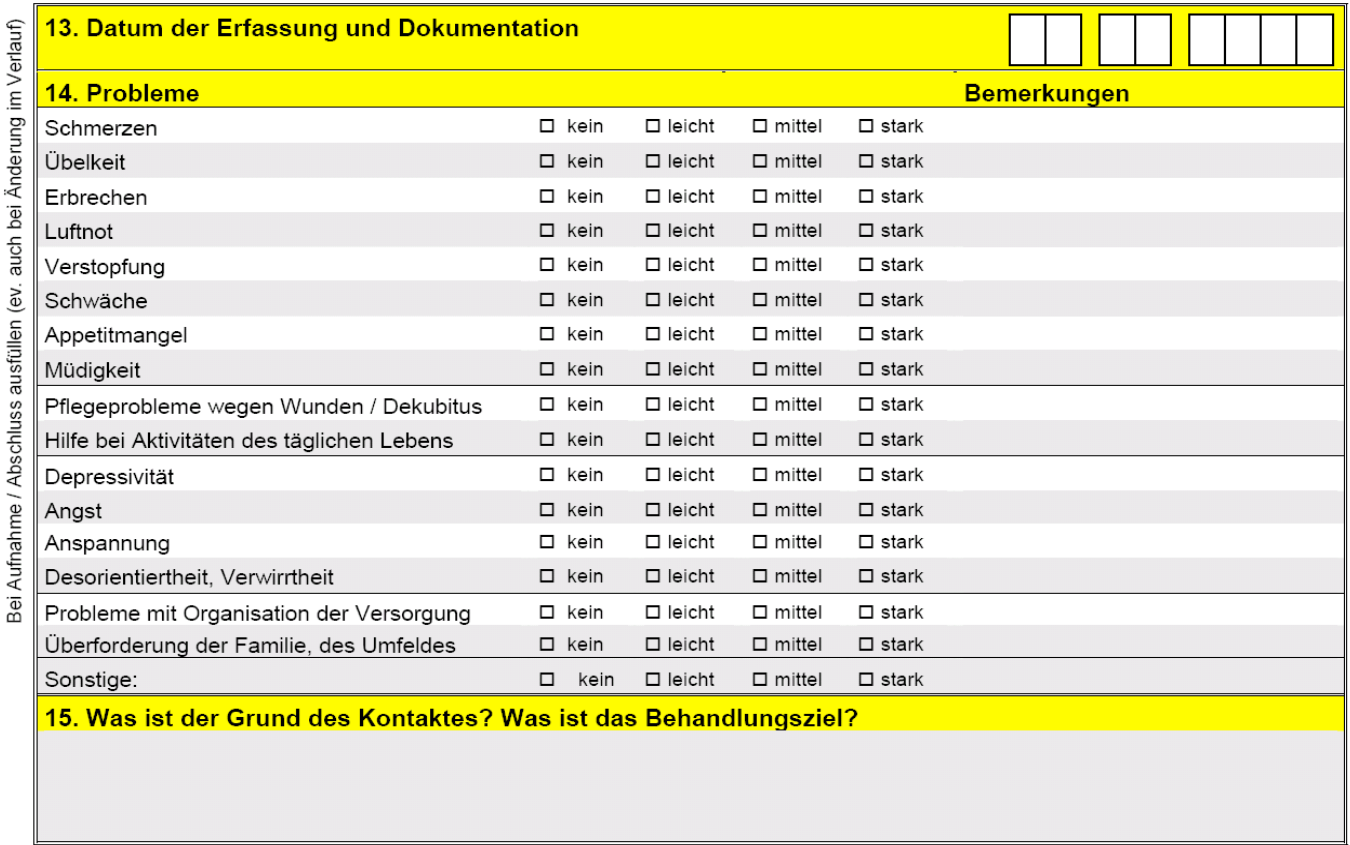




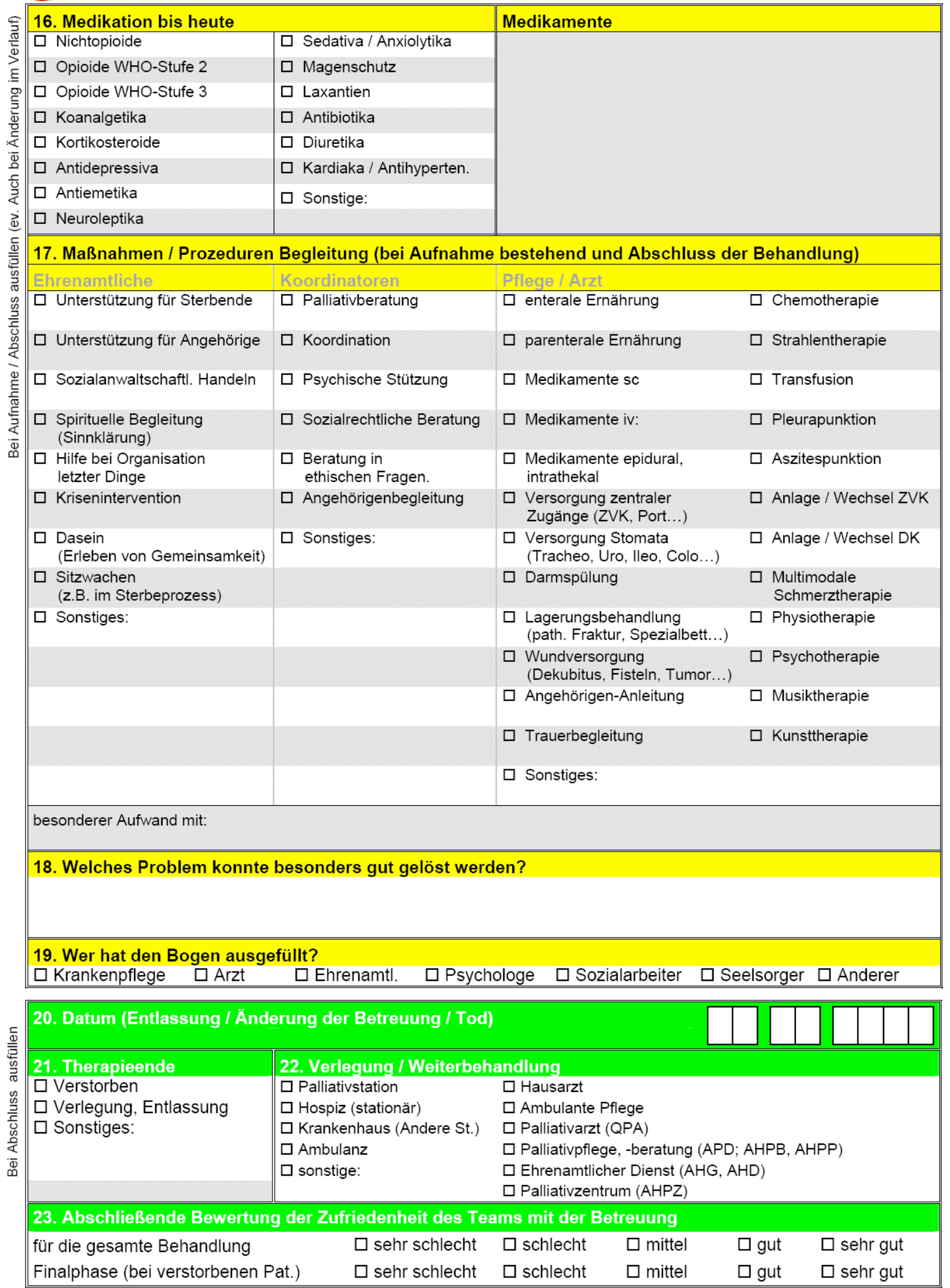




\subsubsection{Hospiz- und Palliativerhebung (HOPE) Modul Diagnostik}

\section{HOPE Q2007 Erweiterte Diagnostik in der Palliativmedizin DI}

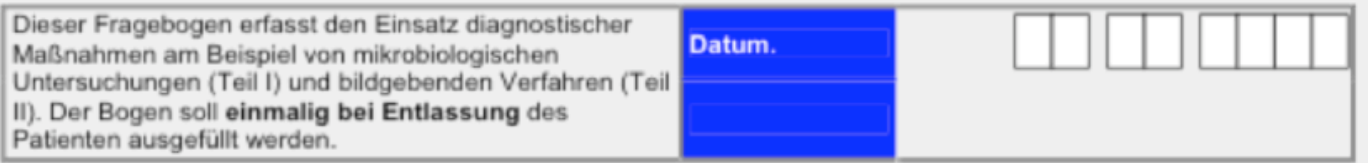

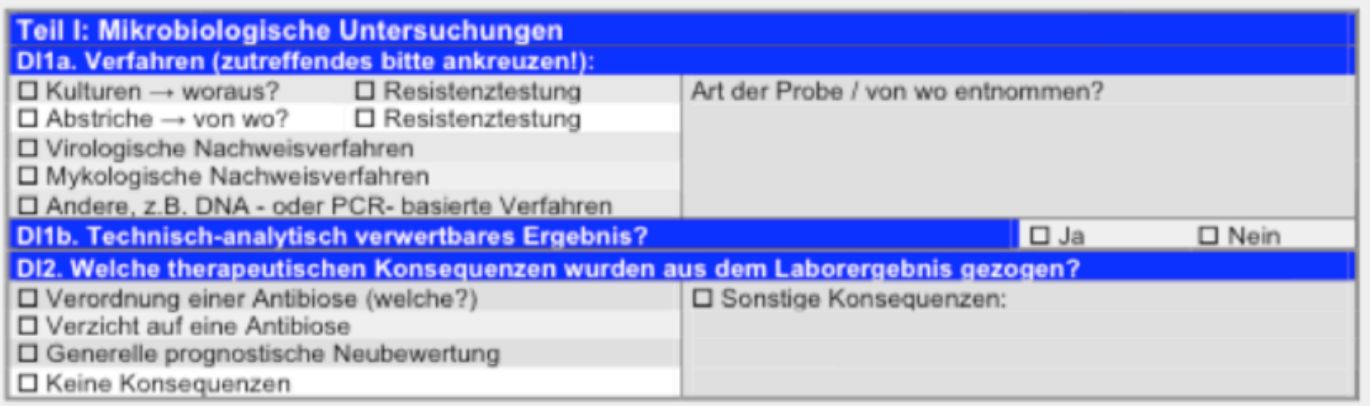

\section{Teil II: Bildgebende Verfahren}

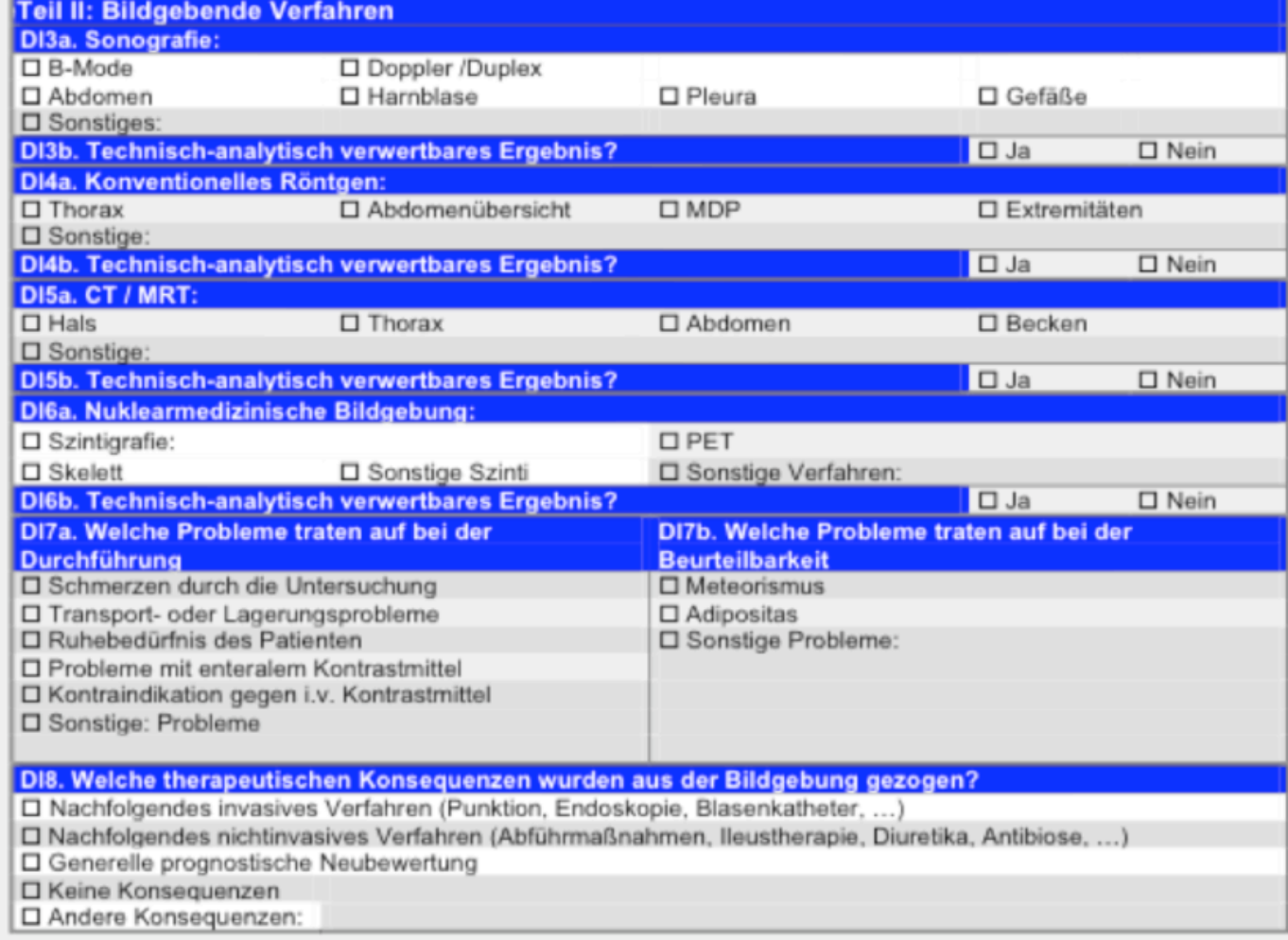

\section{D19. Wer fülit aus?}

Arzt / Arztin 


\subsection{Hospiz- und Palliativerhebung (HOPE) Patienteninformation und Einverständniserklärung}

D) Sehr geehrte Patientinnen und Patienten,

Der Sie betreuende Pflegedienst beteiligt sich an einer Qualitätssicherungsmaßnahme Dieses Projekt wird an mehr als 50 Palliativstationen und Hospizen, mit onkologischen Abteilungen sowie palliativärztlichen und pflegerischen ambulanten Diensten durchgeführt. Grundlage der Qualitätssicherung ist die Dokumentation von Symptomen und Problemen, deren Behandlung und den Erfolgen der Behandlung. Die Dokumentation geschieht in einem einheitlichen Formular (HOPE), das als neuer Standard für Palliativeinrichtungen entwickelt wurde und die wichtigsten Dinge erfasst. Das Formular besteht aus mehreren Teilen für die Ärzte und Schwestern und aus einem Fragebogen für Sie, die Patientinnen und Patienten. In diesem Fragebogen sollen Sie selber über Ihre Beschwerden (z. B. Schmerzen) und Ihr Befinden Auskunft geben. Denn niemand kennt Inre Beschwerden und Ihre Situation so gut wie Sie selbst.
Der Fragebogen wurde für die speziellen Belange der Palliativmedizin entwickelt. Wir möchten Sie bitten, den Fragebogen so sorgfältig und vollständig wie möglich auszufüllen, auch wenn einzelne Fragen für Ihre Situation eventuell nicht zutreffend sind. Wenn Sie einzelne Fragen oder den ganzen Fragebogen nicht beantworten, wird das natürlich keine Nachteile für Sie oder für Ihre weitere Behandlung haben

Die in diesem Fragebogen erhobenen Daten werden mittels eines EDV-Systems gespeichert und anonym (also ohne Namen) ausgewertet. Alle Daten unterliegen selbstverständlich der ärztlichen Schweigepflicht gemäß § 203 StGB und werden nach den Bestimmungen des Bundesdatenschutzes vor jedem Missbrauch geschützt.

Wir danken Innen für Ihre Mitarbeit!

Die Koordinationsgruppe von HOPE 2007
Einverständniserklärung

Ich bin durch die betreuende Person über die

Fragebogenerhebung aufgeklärt worden und habe keine weiteren Fragen.

Ich bin mit der Speicherung der in diesem Fragebogen erhobenen Daten in anonymisierter Form in einem EDV-Dokumentationssystem einverstanden. Sollte ich zu irgendeinem Zeitpunkt mein Einverständnis zurückziehen, werden meine Daten gelöscht.

Datum:

Unterschrift des Patienten: 


\subsection{Tabellen}

\subsubsection{Tabelle von der Homepage der DGP- Kurzinformationen HOPE 2008}

\begin{tabular}{|c|c|c|c|c|}
\hline \multirow[t]{2}{*}{ Jahr } & \multicolumn{2}{|c|}{ Einrichtungen } & \multicolumn{2}{|c|}{ Patienten-Dokumentationen } \\
\hline & Art & Anzahl & & \\
\hline $1999-2001$ & PS & 134 & & 3147 \\
\hline \multirow[t]{4}{*}{2002} & PS & 54 & 1.230 & \\
\hline & OS & 5 & 110 & \\
\hline & G & 2 & 49 & \\
\hline & $\mathrm{H}$ & 8 & 157 & 1.546 \\
\hline \multirow[t]{5}{*}{2004} & PS & 68 & 1.665 & \\
\hline & OS & 3 & 85 & \\
\hline & $\mathrm{H}$ & 12 & 224 & \\
\hline & AA & 7 & 162 & \\
\hline & AP & 4 & 78 & 2.214 \\
\hline \multirow[t]{5}{*}{2005} & PS & 53 & 1.268 & \\
\hline & OS & 6 & 82 & \\
\hline & $\mathrm{H}$ & 12 & 274 & \\
\hline & AA & 8 & 169 & \\
\hline & AP & 5 & 110 & 1.903 \\
\hline \multirow[t]{5}{*}{2006} & PS & 58 & 1.472 & \\
\hline & OS & 7 & 187 & \\
\hline & $\mathrm{H}$ & 22 & 457 & \\
\hline & AA & 29 & 474 & \\
\hline & AP & 15 & 251 & 2.841 \\
\hline \multirow[t]{5}{*}{2007} & PS & 67 & 1.663 & \\
\hline & OS & 8 & 143 & \\
\hline & $\mathrm{H}$ & 24 & 498 & \\
\hline & AA & 28 & 435 & \\
\hline & AP & 22 & 445 & 3.184 \\
\hline \multirow[t]{6}{*}{2008} & PS & 51 & 1.416 & \\
\hline & OS & 42 & 42 & \\
\hline & $\mathrm{H}$ & 9 & 225 & \\
\hline & AA & 7 & 152 & \\
\hline & $\mathrm{K}$ & 5 & 77 & \\
\hline & $\mathrm{AT}$ & 8 & 108 & 2.148 \\
\hline Gesamt & & & & 17.173 \\
\hline
\end{tabular}

Tabelle 9: Teilnehmende Einrichtungen und Anzahl der dokumentierten Patienten in HOPE im Zeitraum 1999- 2008

$\mathrm{PS}=$ Palliativstation, $\mathrm{OS}=$ Onkologische Station, $\mathrm{G}=$ Geriatrische Station, $\mathrm{H}=$ stationäres Hospiz, $\mathrm{AA}=$ ambulanter Arzt, AP = ambulante Pflege, $\mathrm{K}=$ Konsiliardienst, AT = Ambulantes Team 


\subsubsection{Frequenzen der Sonographie im hausärztlichen Bereich}

Frequenzen der Sonographie im hausärztlichen Bereich

\begin{tabular}{|c|c|c|c|c|c|c|}
\hline & GO-Nr. & Beschreibung & $1 / 2008$ & 2/2008 & 3/2008 & 4/2008 \\
\hline \multirow[t]{31}{*}{ ambulant } & 01722 & 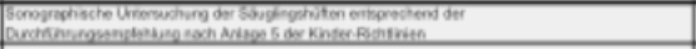 & 9.309 & 9.841 & 10.342 & 9.577 \\
\hline & 33010 & 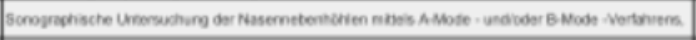 & 413 & 271 & 244 & 293 \\
\hline & 33011 & 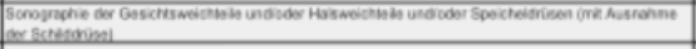 & 1.071 & 1.181 & 1.360 & 1.770 \\
\hline & 33012 & 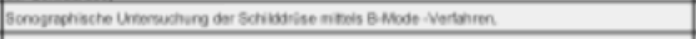 & 48.480 & 52.319 & 43.853 & 44.826 \\
\hline & 33020 & 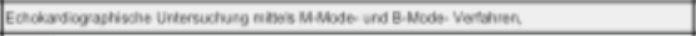 & 563 & 578 & 544 & 488 \\
\hline & 33021 & 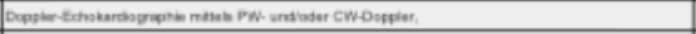 & 68 & 41 & 53 & 43 \\
\hline & 33022 & 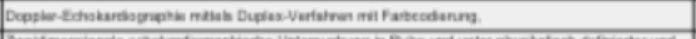 & 985 & 885 & 780 & 807 \\
\hline & 33030 & 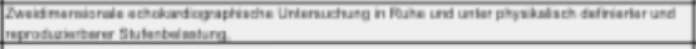 & 1 & 1 & 2 & 1 \\
\hline & 33040 & 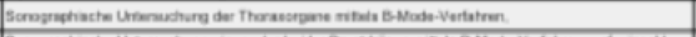 & 3.514 & 3.465 & 3.038 & 3.618 \\
\hline & 33041 & 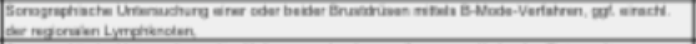 & 19 & 17 & 19 & 21 \\
\hline & 33042 & 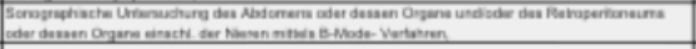 & 262.570 & 255.906 & 225.738 & 234.589 \\
\hline & 33043 & 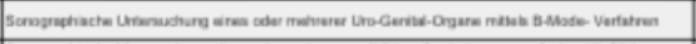 & 4.647 & 4.529 & 4.325 & 4.289 \\
\hline & 33044 & 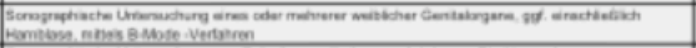 & 1.258 & 1.270 & 1.224 & 1.255 \\
\hline & 33050 & 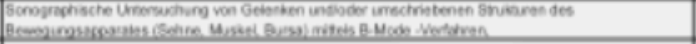 & 2.885 & 2.856 & 2.939 & 2.472 \\
\hline & 33051 & 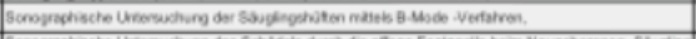 & 4.124 & 4.206 & 4.077 & 3.913 \\
\hline & 33052 & 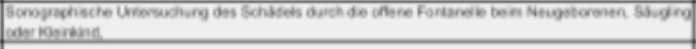 & 1.085 & 1.146 & 1.054 & 1.029 \\
\hline & 33060 & 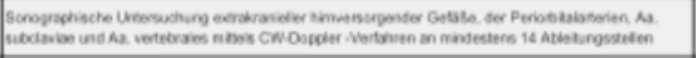 & 2.084 & 2.089 & 1.772 & 2.165 \\
\hline & 33061 & 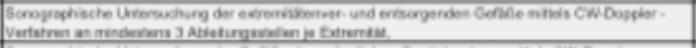 & 8.615 & 8.866 & 7.525 & 8.964 \\
\hline & 33062 & 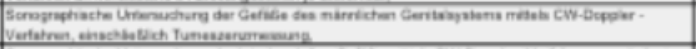 & & & & 1 \\
\hline & 33063 & 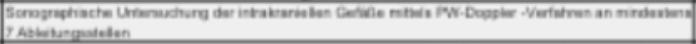 & 6 & 8 & 11 & 8 \\
\hline & 33070 & 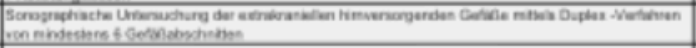 & 288 & 231 & 165 & 299 \\
\hline & 33071 & 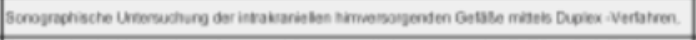 & 1 & 8 & 3 & 3 \\
\hline & 33072 & 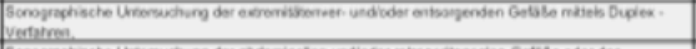 & 1.074 & 1.216 & 1.244 & 419 \\
\hline & 33073 & 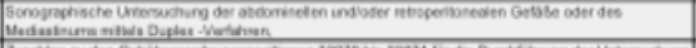 & 4.237 & 4.806 & 4.498 & 4.302 \\
\hline & 33075 & 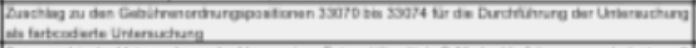 & 5.174 & 6.168 & 5.833 & 4.884 \\
\hline & 33076 & 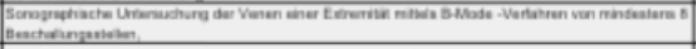 & 2.207 & 2.733 & 2.700 & 2.506 \\
\hline & 33080 & 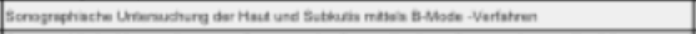 & & & 1 & 1 \\
\hline & 33081 & 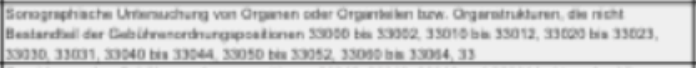 & 949 & 1.011 & 1.034 & 1.206 \\
\hline & 33090 & 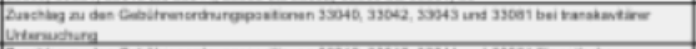 & 183 & 160 & 182 & 232 \\
\hline & 33091 & 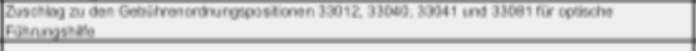 & 4 & 29 & 9 & 5 \\
\hline & 33092 & 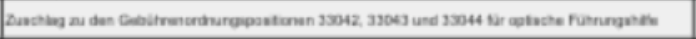 & 136 & 91 & 136 & 98 \\
\hline \multicolumn{3}{|c|}{ Summe ambulanter Bereich } & 365.950 & 365.928 & 324.705 & 334.084 \\
\hline \multirow[t]{6}{*}{ stationär } & 01722 & 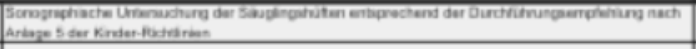 & 1 & & & \\
\hline & 33012 & 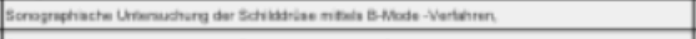 & & & 1 & \\
\hline & 33040 & 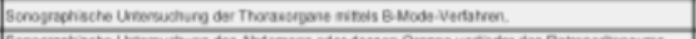 & 3. & 2. & & 1 \\
\hline & 33042 & 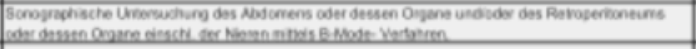 & 44 & 42 & 38 & 40 \\
\hline & 33051 & 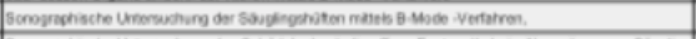 & 4 & 7 & 5 & 4 \\
\hline & 33052 & 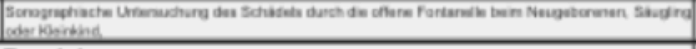 & 12 & 8 & 15 & 10 \\
\hline \multicolumn{3}{|c|}{ Summe stationärer Bereich } & 64 & 59 & 59 & 55 \\
\hline \multicolumn{3}{|c|}{ Gesamtergebnis } & 366.014 & 365.987 & 324.764 & 334.139 \\
\hline
\end{tabular}




\subsection{Dokumentationsbogen der UMG Patienten}

\section{Sonographie}

Patientennummer:

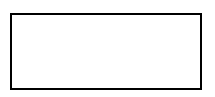

Datum der Untersuchung:

Geburtsdatum:

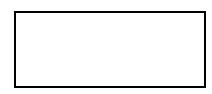

Geschlecht:

weibl.

männl.

Aufnahmedatum:

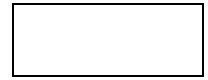

Entlassungsdatum:
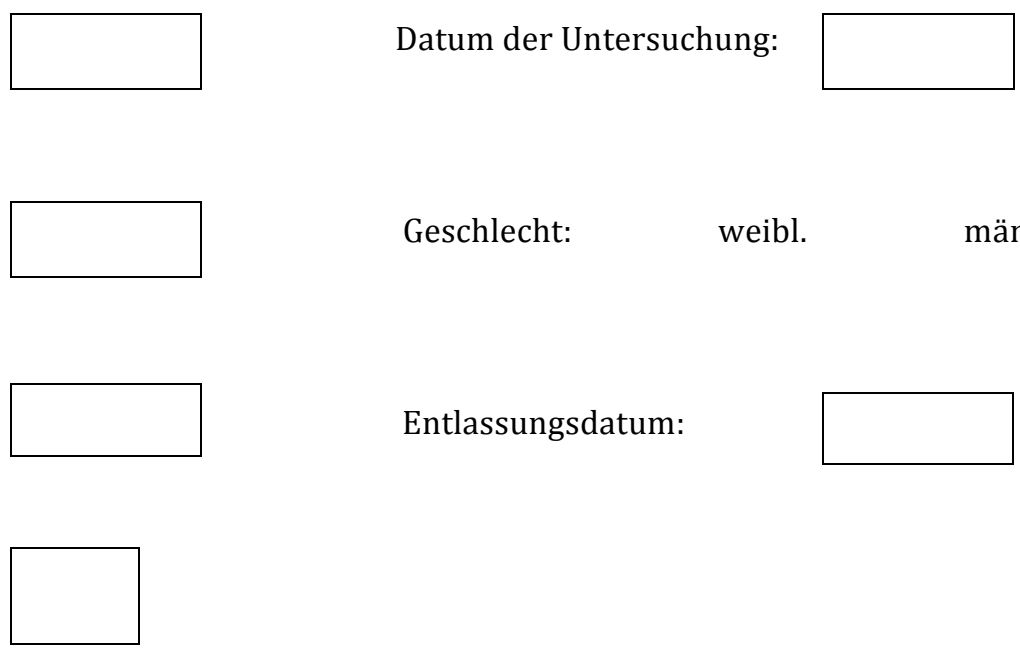

Indikation/ Fragestellung:

Untersuchte Organe:

\begin{tabular}{|c|c|c|c|}
\hline Hals & & Milz & \\
\hline Pleura & & Niere re. & \\
\hline Leber & & Niere li. & \\
\hline Gallenblase & & Harnblase & \\
\hline Gallenwege & & Prostata & \\
\hline Pankreas & & Art. Abdominalis & \\
\hline Darm & & V. cava & \\
\hline LK & & Pfortader & \\
\hline
\end{tabular}

Technisch/ analytisches Ergebnis: Ja Nein

Therapeutische Konsequenzen:
A Symptomabklärung/ Verlaufskontrolle ohne weiteren Konsequenzen im Bezug auf die aktuelle Therapie
B Nachfolgendes invasives Verfahren ( Punktion, Endoskopie, Blasenkatheter...)
C Nachfolgendes NICHT invasives Verfahren (Abführmaßnahmen, Ileustherapie, Antibiose, Diuretika,....)
D Prognostische Neubewertung mit Abbruch der Therapie
E Prognostische Neubewertung Änderung/ oder neue Therapie
F Konsil 


\subsection{Englischsprachige Definition „Palliative care“ gemäß WHO (http://www.who.int/cancer/palliative/definition/en/ 2009):}

Palliative Care is an approach that improves the quality of live of patients and their families facing the problem associated with life-threatening illness, through the prevention and relief of suffering by means of early identification and impeccable assessment and treatment of pain and other problems, physical, psychosocial and spiritual. Palliative care:

- Provides relief from pain and other distressing symptoms;

- Affirms life and regards dying as a normal process;

- Intends neither to hasten or postpone death;

- Integrates the psychological and spiritual aspects of patient care;

- Offers a support system to help patients live as actively as possible until death;

- Offers a support system to help the family cope during the patient 's illness and in their own bereavement;

- Uses a team approach to address the needs of patients and their families, including bereavement counseling, if indicated;

- Will enhance quality of live, and may also positively influence the course of illness;

- Is applicable early in the course of illness, in conjunction with other therapies that

- are intended to prolong life, such as chemotherapy or radiation therapy, and includes those investigations needed to better understand and manage distressing clinical complications. 


\section{Literaturverzeichnis}

André B, Ringdal G I, Loge J H, Rannestad T, Kaasa S (2009): Implementation of computerized technology in a palliative care unit. J Palliat and Supp Care 7, 57-63

Aulbert E: Ganzheitliche Symptomkontrolle als Basis der Palliativmedizin. In: Band 1: Palliativmedizin- Ein ganzheitliches Konzept; hrsg v. Aulbert E, Klaschik E, Pichlmaier H: Schattauer Verlag, Stuttgart 1998, 25-36

Aulbert E, Klaschik E, Pichlmaier H: Palliativmedizin - Die Alternative zur aktiven Sterbehilfe. Schattauer Verlag, Stuttgart 1998, 54

Aulbert E, Nauck F, Radbruch L: Lehrbuch der Palliativmedizin. 2. vollst. überarb. u. erw. Auflage: Schattauer Verlag, Stuttgart 2008

Bausewein C, Fegg M, Radbruch L, Nauck F, von Mackensen S, Borasio GD, Higginson IJ (2005): Validation and Clinical Application of the German Version of the Palliative Care Outcome Scale. J Pain Symptom Manage, 30(1), 51-62

Bausewein C, Roller S, Voltz R: Hospiz und PalliativErhebung (HOPE) in: Leitfaden Palliativmedizin. 3. Auflage: Urban\& Fischer Verlag, München 2004, 53

Becker-Schwarze K: Die palliativmedizinische Behandlung als ärztlich geschuldete Leistung. In: Recht und Ethik der Palliativmedizin. Hrsg. v Höfling W, Brysch E: LIT Verlag, Berlin-Hamburg-Münster 2007, 37-52

Conill C, Verger E, Henríquez I, Saiz N, Espier M, Lugo F, Garrigos A (1997): Symptom prevalence in the last week of life. J Pain Symptom Manage. 14, 328-31

Department of health (2007): Link: http://www.dh.gov.uk/en/index.htm (01.07.2007)

Deutsche Gesellschaft für Palliativmedizin (2003): Definition Palliativmedizin 31.10.2003, www.dgpalliativmedizin.de

Deutsche Gesellschaft für Palliativmedizin (DGP) (2006): Links: Ausbildungsinstitute. http://www.dgpalliativmedizin.de (5. 6. 2006) 
Deutsche Gesellschaft für Palliativmedizin (2007): Link: www.dgpalliativmedizin.de (01.07.2007)

Dietz I, Laske A: Entwicklung der Palliativmedizinischen Lehre in Deutschland 2009, Poster 11. Kongress der EAPC, Mai 2009, AG Palliativmedizin www.pallmed.de

Doyle D, Hanks G, MacDonald N (Hrsg): Oxford Textbook of Palliative Medicine. 3.Auflage: Oxford University Press, New York 2005, 1-46

European Association for Palliative Care (2004): Definition of Palliative Care. www.eapenet.org/about/definition.html (29.09.2004)

Gebel MJ (2000): New procedures and modifications of sonographyand their practical significane. Internist, $41(1), 3-9$

Gehmacher 0 (2006): Symptomorientiertes Ultraschallen bei Palliativpatienten. Z Pall 7, $137-141$

Gishen F, Trotman J (2009): Bedside ultrasound-experience in palliative care units. J Cancer Care, 18(6), 642-4

Grond S, Zech D, Diefenbach C, Bischoff A (1994): Prevalence and pattern of Symptoms in patients with cancer pain: a prospective evaluation of 1635 cancer patients referred to a pain clinic. J Pain Symptom Manage. 9, 372-82

Harstenstein R (2002): Stellenwert der Palliativmedizin in der Onkologie. Onkologie, 25 (Suppl 1), 60-64

Homsi J, Walsch D, Panta R, Lagman R, Nelson KA, Longworth DL (2000): Infectious complications of advanced cancer. Support Care Cancer. 8(6), 487-92

Jonen-Thielemann I: Definition der letzten Lebensphasen. In: Aulbert E, Nauck F, Radbruch L, Lehrbuch der Palliativmedizin. 2. Auflage: Schattauer Verlag, Stuttgart 2007, 1020-28.

Jordhoy MS, Kaasa S, Fayers P, Ovreness T, UnderlandG, Ahlner-Elmqvist M. (1999): Challenges in palliative care research; recruitment, attrition and compliance: experience from a randomised controlled trial. Palliat Med, 13, 299-310. 
Kassenärztliche Bundesvereinigung (KVB) (2009): Links: Themen. http://www.kbv.de/Ultraschall.html (25.08.2009)

Klaschik E (2001): Palliativmedizin- Ganzheitliche Medizin mit hohem Entwicklungsbedarf. Schmerz, 15, 311

Klaschik E, Nauck F, Kern M (1998): Palliativmedizin. Z Arztl Fortbild Qualitatssich, $\underline{92,53}$

Klaschik E, Nauck F, Radbruch L, Sabalowski R (2000): Palliativmedizin- Definition und Grundzüge. Internist, $\underline{41}$, 606- 611

Klinkhammer G (2007): Palliativmedizin- Junge Disziplin mit großem Potential. Dtsch Arztebl, 5, 217-220

Lang K, Puhlmann K, Falckenberg M (2006): Aus-, Fort- und Weiterbildung in der Palliativversorgung. Bundesgesundheitsblatt Gesundheitsforschung Gesundheitsschutz $\underline{49(11), 1149-54}$

Lam PT, Chan KS, Tse CY, Leugen MW (2005): Retrospective analysis of antibiotic use and survival in advanced cancer patients with infections. J Pain Symptom Manage. $30(6)$, 536-43.

Lindena G, Nauck F, Bausewein C, Neuwohner K, Heine O, Schulenberg D, Radbruch L (2005): Qualitätssicherung in der Palliativmedizin - Ergebnisse der Kerndokumentation 1999-2002. Z Arztl Fortbild Qual Gesundh wes 99, 555-65

Ling J, Rees E, Hardy J. (2000): What influences participation in clinical trials in palliative care in a cancer centre? Eur J Cancer, 36, 621-26

Mariani P, Setlaj A (2010): Palliative ultrasound for home care hospice patients. Acad Emerg Med, 17(3), 293-6

Maschmeyer G: Infektionen bei neutropenischen Patienten In: Therapie innerer Krankheiten. 11. Auflage: hrsg. v Paumgartner G, Steinbeck G: Springer Verlag, Heidelberg 2005, 1672-79 
Müller M, Kern M, Nauck F, Klaschik E: Quallifikation hauptamtlicher Mitarbeiter. Curricula für Ärzte, Pflegende, Sozialarbeiter und Seelsorger in Palliativmedizin. Pallia Med Verlag, Bonn 1997

Nagy-Agren S, Haley HB (2002): Management of infections in palliative care patients with advanced cancer. J Pain Syndrom Manage, 24 (1), 64-70

Nauck F (2009): Neues Querschnittsfach- Palliativmedizin nun Pflichtlehr- und $\begin{array}{lll}\text { Prüfungsfach. Niedersächs } \quad \text { Ärztebl, } & \text { 10/2009. }\end{array}$ http://www.haeverlag.de/nae/n_beitrag.php?id=2746

Nauck F, Radbruch L (2002): Palliativmedizin in NRW- Gegenwärtiger Stand und Notwendigkeiten der zukünftigen Entwicklung. Rhein Ärztebl, 2, 11-14

Nauck F, Fittkau-Tönnesmann B, Kern M : Aus-, Fort- und Weiterbildung in der Palliativmedizin. In: Lehrbuch der Palliativmedizin. 2. vollst. überarb. u. erw. Auflage: hrsg. v Aulbert E, Nauck F, Radbruch L: Schattauer Verlag, Stuttgart 2008, 1309-17

Pache S: Persönliche Mittelung. Göttingen 2010

Pereira J, Watanabe S, Wolch G (1998): A retrospective review of the frequency of infections and patterns of antibiotic utilization on a palliative care unit. J Pain Symptom Manage, 16(6), 374-81

Radbruch L, Nauck F (2008): Patientenregister als Forschungsinstrument- am Beispiel der Hospiz- und Palliativerhebnung (HOPE). www.dgpalliativmedizin.de/agforhope.html

Radbruch L, Sabatowski R, Loick G, Jonen- Thielemann I, Elsner F, Hörmann E (2000): MIDOS- Validierung eines minimalen Dokumentationssystems für die Palliativmedizin. Schmerz, 14, 231-239

Radbruch L, Nauck F, Fuchs M, Neuwohner K, Schulenberg D, Lindena G (2002): What is palliative care in Germany? Results from a representative survey. J Pain Symptom Manage 23(6), 471-83 
Radbruch L, Nauck F, Ostgathe C, Elsner F, Bausewein C, Fuchs M, Lindena G, Neuwöhner K, Schulenberg D (2003): What are the problems in palliative care? Results from a representative survey. Support Care Cancer, 11, 442-451

Reinbolt R.E, Shenk AM, With PH, Navari RM (2005): Symptomatic treatment of infections in patients with advanced cancer receiving hospice care. J Pain Symptom Manage. 30(2), 175-82

Ripamonti C, Fusco F (2002): Respiratory problems in advanced cancer. Support Care Cancer. 10(3), 204-16

Röglin H-C (1998): Menschenwürdiger Umgang mit dem Sterben aus Sicht praktizierender Onkologen.Forum DKG 13, 244-252

Sabatowski R, Radbruch L, Nauck F, Loick G, Meuser T, Lehmann KA (2001): Entwicklung und Stand der stationären palliativmedizinischen Einrichtungen in Deutschland. Schmerz, 15, 312- 319

Samonigg H, Kasparek A K, Andritsch E (2000): Ganzheitliche Tumorpatientenbetreuung in der letzten Lebensphase. Onkologe 6 , 533-539

Saunders C (1977): On dying and dying well. Proc R Soc Med, 70(4), 290-1

Sorbye LW (2000): A longitudinal study on dying in an Norwegian hospital. J Palliat Nurs, $6(2), 71-79$

Twycross R.G. (1980): Hospice care - redressing the balance in medicine. J R Soc Med, 73, 827-835

Vitetta, L, Kenner D, Sali A (2000): Bacterial infections in terminally ill hospice patients. J Pain Symptom Manage 20(5), 326-34

World Health Organization: Definition Palliativmedizin http://www.who.int/cancer/palliative/definition/en/ 03.09.2009) 


\section{Veröffentlichungen}

Teile der Ergebnisse dieser Dissertation wurden auf folgenden medizinischwissenschaftlichen Fachkongressen vorgestellt:

\section{Als Vortrag:}

Diagnostik in der Palliativmedizin, 23.02.2008, 28. Deutscher Krebskongress vom 20.23. Februar 2008 in Berlin (Referentin: Ruth Sürig, geb. Baumann)

Wie viel Diagnostik verträgt die Palliativmedizin? 26.9.2008, 7. Kongress der Deutschen Gesellschaft für Palliativmedizin vom 25.-27.September 2008 in Wiesbaden

\section{Als Abstract:}

7. Kongress der Deutschen Gesellschaft für Palliativmedizin vom 25.-27.September 2008 in Wiesbaden

Alt-Epping B, Baumann R, Lindena G, Nauck F: Ausmaß und therapeutische Relevanz nicht-invasiver Diagnostik in der Palliativmedizin. Vortrag FV 014, Kongress der DGP Wiesbaden 2008

\section{Als Poster:}

11th Congress of the European Association for Palliative Care (EAPC) Vienna (Austria) 2009

Baumann RM, Alt-Epping B, Lindena G, Nauck F. Therapeutic relevance of non-invasive diagnostics in palliative care - results from a representative survey and own experiences. 


\section{Danksagung}

Ich möchte mich bei allen bedanken, die mir bei der Entstehung dieser Arbeit zur Seite gestanden haben. An erster Stelle gilt mein Dank Herrn Prof. Dr. med. F. Nauck für die Bereitstellung des Themas, für seine unkomplizierte Art der Unterstützung, seine aufmunternden und herzlichen Worte sowie seine konstruktiven Verbesserungsvorschläge.

Mein besonderer Dank gilt Herrn Dr. med. B. Alt-Epping für seine unendliche Geduld, seine stets ausgezeichnete Betreuung, seine umfangreiche Beratung sowie seinen Beistand und seine Hilfe bei auftretenden Problemen.

Frau Dr. Lindena von CLARA (Clinical Analysis, Research and Application) danke ich herzlich für die Überlassung der Daten. Nicht unerwähnt lassen möchte ich auch einen Dank an alle Einrichtungen, die an HOPE 2007 teilgenommen haben, den 70 Patienten und den Mitarbeitern der Palliativstation Göttingen.

Für die statistische Betreuung meiner Arbeit gilt mein großer Dank der Abteilung Medizinische Statistik / Epidemiologie der UMG und deren Mitarbeitern.

Besonderer Dank gilt außerdem meiner Mutter für das Korrekturlesen, für Kritik, Auseinandersetzung und Ermutigung während der Entstehung dieser Arbeit. 


\section{Lebenslauf}

Mein Name ist Ruth Maria Sürig, geb. Baumann, wohnhaft Georg-Eckert-Strasse 5A in 38100 Braunschweig. Am 11. Dezember 1981 wurde ich als erstes Kind von Marga Maria Baumann, geb. Pley, (Realschullehrerin) und Walter Baumann (Diplom-Ingenieur) in Düren geboren. Ich habe einen Bruder Jörg Paul Jakob Baumann (24 Jahre).

Von 1987-1991 besuchte ich die Gemeinschaftsgrundschule Merken, anschließend wechselte ich auf das Gymnasium am Wirteltor in Düren (1991-2001), an dem ich im Jahre 2001 erfolgreich mein bilinguales Abitur ablegte.

Zum Wintersemester 2001/02 nahm ich das Studium der Humanmedizin an der GeorgAugust-Universität in Göttingen auf. Dort bestand ich im September 2005 mein Physikum. Im Anschluss daran, von Oktober 2005 bis März 2006, absolvierte ich ein Praktikum bei einem staatlich anerkannten Physiotherapeuten. Im April 2006 begann ich den klinischen Abschnitt meines Medizinstudiums in Göttingen. Von Februar 2009 bis Februar 2010 absolvierte ich das Praktische Jahr meiner Ausbildung. Das erste Tertial (Innere Medizin) absolvierte ich am St. Ansgar Krankenhaus in Höxter, das zweite Tertial (Wahlfach HNO) am Universitätsklinikum Göttingen. Das dritte Tertial (Chirurgie) habe ich zur Hälfte am Universitätsklinikum in Göttingen und zur Hälfte in Kapstadt (Süd Afrika) am Tygerberg Hospital absolviert. Mein Studium habe ich im Mai 2010 erfolgreich mit dem zweiten Staatsexamen abgeschlossen und arbeite seit September 2010 als Assistenzärztin im Städtischen Klinikum Braunschweig (Abteilung für Hals- Nasen- Ohrenheilkunde).

Ruth Maria Sürig 